\title{
Basic properties of SLE
}

\author{
By Steffen Rohde* and Oded Schramm \\ Dedicated to Christian Pommerenke on the occasion of his 70th birthday
}

\begin{abstract}
$\mathrm{SLE}_{\kappa}$ is a random growth process based on Loewner's equation with driving parameter a one-dimensional Brownian motion running with speed $\kappa$. This process is intimately connected with scaling limits of percolation clusters and with the outer boundary of Brownian motion, and is conjectured to correspond to scaling limits of several other discrete processes in two dimensions.

The present paper attempts a first systematic study of SLE. It is proved that for all $\kappa \neq 8$ the SLE trace is a path; for $\kappa \in[0,4]$ it is a simple path; for $\kappa \in(4,8)$ it is a self-intersecting path; and for $\kappa>8$ it is space-filling.

It is also shown that the Hausdorff dimension of the $\mathrm{SLE}_{\kappa}$ trace is almost surely (a.s.) at most $1+\kappa / 8$ and that the expected number of disks of size $\varepsilon$ needed to cover it inside a bounded set is at least $\varepsilon^{-(1+\kappa / 8)+o(1)}$ for $\kappa \in[0,8)$ along some sequence $\varepsilon \searrow 0$. Similarly, for $\kappa \geq 4$, the Hausdorff dimension of the outer boundary of the $\mathrm{SLE}_{\kappa}$ hull is a.s. at most $1+2 / \kappa$, and the expected number of disks of radius $\varepsilon$ needed to cover it is at least $\varepsilon^{-(1+2 / \kappa)+o(1)}$ for a sequence $\varepsilon \searrow 0$.
\end{abstract}

\section{Introduction}

Stochastic Loewner Evolution (SLE) is a random process of growth of a set $K_{t}$. The evolution of the set over time is described through the normalized conformal map $g_{t}=g_{t}(z)$ from the complement of $K_{t}$. The map $g_{t}$ is the solution of Loewner's differential equation with driving parameter a onedimensional Brownian motion. SLE, or $\mathrm{SLE}_{\kappa}$, has one parameter $\kappa \geq 0$, which is the speed of the Brownian motion. A more complete definition appears in Section 2 below.

The SLE process was introduced in [Sch00]. There, it was shown that under the assumption of the existence and conformal invariance of the scaling limit of loop-erased random walk, the scaling limit is $\mathrm{SLE}_{2}$. (See Figure 9.1.) It was also stated there without proof that $\mathrm{SLE}_{6}$ is the scaling limit of the

*Partially supported by NSF Grants DMS-0201435 and DMS-0244408. 


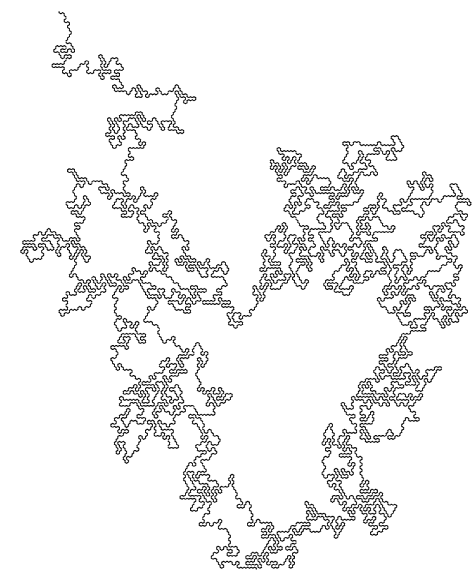

Figure 1.1: The boundary of a percolation cluster in the upper half plane, with appropriate boundary conditions. It converges to the chordal $\mathrm{SLE}_{6}$ trace.

boundaries of critical percolation clusters, assuming their conformal invariance. Smirnov [Smi01] has recently proved the conformal invariance conjecture for critical percolation on the triangular grid and the claim that $\mathrm{SLE}_{6}$ describes the limit. (See Figure 1.1.) With the proper setup, the outer boundary of $\mathrm{SLE}_{6}$ is the same as the outer boundary of planar Brownian motion [LSW03] (see also [Wer01]). $\mathrm{SLE}_{8}$ has been conjectured [Sch00] to be the scaling limit of the uniform spanning tree Peano curve (see Figure 9.2), and there are various further conjectures for other parameters. Most of these conjectures are described in Section 9 below. Also related is the work of Carleson and Makarov [CM01], which studies growth processes motivated by DLA via Loewner's equation.

SLE is amenable to computations. In [Sch00] a few properties of SLE have been derived; in particular, the winding number variance. In the series of papers [LSW01a], [LSW01b], [LSW02], a number of other properties of SLE have been studied. The goal there was not to investigate SLE for its own sake, but rather to use $\mathrm{SLE}_{6}$ as a means for the determination of the Brownian motion intersection exponents.

As the title suggests, the goal of the present paper is to study the fundamental properties of SLE. There are two main variants of SLE, chordal and radial. For simplicity, we concentrate on chordal SLE; however, all the main results of the paper carry over to radial SLE as well. In chordal SLE, the set $K_{t}, t \geq 0$, called the SLE hull, is a subset of the closed upper half plane $\overline{\mathbb{H}}$ and $g_{t}: \mathbb{H} \backslash K_{t} \rightarrow \mathbb{H}$ is the conformal uniformizing map, suitably normalized at infinity.

We show that with the possible exception of $\kappa=8$, a.s. there is a (unique) continuous path $\gamma:[0, \infty) \rightarrow \overline{\mathbb{H}}$ such that for each $t>0$ the set $K_{t}$ is the union of $\gamma[0, t]$ and the bounded connected components of $\overline{\mathbb{H}} \backslash \gamma[0, t]$. The path $\gamma$ is called the SLE trace. It is shown that $\lim _{t \rightarrow \infty}|\gamma(t)|=\infty$ a.s. We also 
describe two phase transitions for the SLE process. In the range $\kappa \in[0,4]$, a.s. $K_{t}=\gamma[0, t]$ for every $t \geq 0$ and $\gamma$ is a simple path. For $\kappa \in(4,8)$ the path $\gamma$ is not a simple path and for every $z \in \mathbb{H}$ a.s. $z \notin \gamma[0, \infty)$ but $z \in \bigcup_{t>0} K_{t}$. Finally, for $\kappa>8$ we have $\overline{\mathbb{H}}=\gamma[0, \infty)$ a.s. The reader may wish to examine Figures 9.1, 1.1 and 9.2, to get an idea of what the $\mathrm{SLE}_{\kappa}$ trace looks like for $\kappa=2,6$ and 8 , respectively.

We also discuss the expected number of disks needed to cover the $\mathrm{SLE}_{\kappa}$ trace and the outer boundary of $K_{t}$. It is proved that the Hausdorff dimension of the trace is a.s. at most $1+\kappa / 8$, and that the Hausdorff dimension of the outer boundary $\partial K_{t}$ is a.s. at most $1+2 / \kappa$ if $\kappa \geq 4$. For $\kappa \in[0,8)$, we also show that the expected number of disks of size $\varepsilon$ needed to cover the trace inside a bounded set is at least $\varepsilon^{-(1+\kappa / 8)+o(1)}$ along some sequence $\varepsilon \searrow 0$. Similarly, for $\kappa \geq 4$, the expected number of disks of radius $\varepsilon$ needed to cover the outer boundary is at least $\varepsilon^{-(1+2 / \kappa)+o(1)}$ for a sequence of $\varepsilon \searrow 0$. Richard Kenyon has earlier made the conjecture that the Hausdorff dimension of the outer boundary is a.s. $1+2 / \kappa$. These results offer strong support for this conjecture.

It is interesting to compare our results to recent results for the deterministic Loewner evolution, i.e., the solutions to the Loewner equation with a deterministic driving function $\xi(t)$. In $[\mathrm{MR}]$ it is shown that if $\xi$ is Hölder continuous with exponent $1 / 2$ and small norm, then $K_{t}$ is a simple path. On the other hand, there is a function $\xi$, Hölder continuous with exponent $1 / 2$ and having large norm, such that $K_{t}$ is not even locally connected, and therefore there is no continuous path $\gamma$ generating $K_{t}$. In this example, $K_{t}$ spirals infinitely often around a disk $D$, accumulating on $\partial D$, and then spirals out again. It is easy to see that the disk $D$ can be replaced by any compact connected subset of $\mathbb{H}$. Notice that according to the law of the iterated logarithm, a.s. Brownian motion is not Hölder continuous with exponent $1 / 2$. Therefore, it seems unlikely that the results of the present paper can be obtained from deterministic results.

Our results are based on the computation and estimates of the distribution of $\left|g_{t}^{\prime}(z)\right|$ where $z \in \mathbb{H}$. Note that in [LSW01b] the derivatives $g_{t}^{\prime}(x)$ are studied for $x \in \mathbb{R}$.

The organization of the paper is as follows. Section 2 introduces the basic definitions and some fundamental properties. The goal of Section 3 is to obtain estimates for quantities related to $\mathbf{E}\left[\left|g_{t}^{\prime}(z)\right|^{a}\right]$, for various constants $a$ (another result of this nature is Lemma 6.3), and to derive some resulting continuity properties of $g_{t}^{-1}$. Section 4 proves a general criterion for the existence of a continuous trace, which does not involve randomness. The proof that the $\mathrm{SLE}_{\kappa}$ trace is continuous for $\kappa \neq 8$ is then completed in Section 5. There, it is also proved that $g_{t}^{-1}$ is a.s. Hölder continuous when $\kappa \neq 4$. Section 6 discusses the two phase transitions $\kappa=4$ and $\kappa=8$ for $\mathrm{SLE}_{\kappa}$. Besides some quantitative 
properties, it is shown there that the trace is a.s. a simple path if and only if $\kappa \in[0,4]$, and that the trace is space-filling for $\kappa>8$. The trace is proved to be transient when $\kappa \neq 8$ in Section 7. Estimates for the dimensions of the trace and the boundary of the hull are established in Section 8. Finally, a collection of open problems is presented in Section 9.

Update. Since the completion and distribution of the first version of this paper, there has been some further progress. In [LSW] it was proven that the scaling limit of loop-erased random walk is $\mathrm{SLE}_{2}$ and the scaling limit of the UST Peano path is $\mathrm{SLE}_{8}$. As a corollary of the convergence of the UST Peano path to $\mathrm{SLE}_{8}$, it was also established there that $\mathrm{SLE}_{8}$ is generated by a continuous transient path, thus answering some of the issues left open in the current paper. However, it is quite natural to ask for a more direct analytic proof of these properties of $\mathrm{SLE}_{8}$.

Recently, Vincent Beffara [Bef] has announced a proof that the Hausdorff dimension of the $\mathrm{SLE}_{\kappa}$ trace is $1+\kappa / 8$ when $4 \neq \kappa \leq 8$.

The paper $[\mathrm{SS}]$ proves the convergence of the harmonic explorer to $\mathrm{SLE}_{4}$.

\section{Definitions and background}

2.1. Chordal SLE. Let $B_{t}$ be Brownian motion on $\mathbb{R}$, started from $B_{0}=0$. For $\kappa \geq 0$ let $\xi(t):=\sqrt{\kappa} B_{t}$ and for each $z \in \overline{\mathbb{H}} \backslash\{0\}$ let $g_{t}(z)$ be the solution of the ordinary differential equation

$$
\partial_{t} g_{t}(z)=\frac{2}{g_{t}(z)-\xi(t)}, \quad g_{0}(z)=z .
$$

The solution exists as long as $g_{t}(z)-\xi(t)$ is bounded away from zero. We denote by $\tau(z)$ the first time $\tau$ such that 0 is a limit point of $g_{t}(z)-\xi(t)$ as $t \nearrow \tau$. Set

$$
H_{t}:=\{z \in \mathbb{H}: \tau(z)>t\}, \quad K_{t}:=\{z \in \overline{\mathbb{H}}: \tau(z) \leq t\} .
$$

It is immediate to verify that $K_{t}$ is compact and $H_{t}$ is open for all $t$. The parametrized collection of maps $\left(g_{t}: t \geq 0\right)$ is called chordal $\mathrm{SLE}_{\kappa}$. The sets $K_{t}$ are the hulls of the SLE. It is easy to verify that for every $t \geq 0$ the map $g_{t}: H_{t} \rightarrow \mathbb{H}$ is a conformal homeomorphism and that $H_{t}$ is the unbounded component of $\mathbb{H} \backslash K_{t}$. The inverse of $g_{t}$ is obtained by flowing backwards from any point $w \in \mathbb{H}$ according to the equation (2.1). (That is, the fact that $g_{t}$ is invertible is a particular case of a general result on solutions of ODE's.) One only needs to note that in this backward flow, the imaginary part increases, hence the point cannot hit the singularity. It also cannot escape to infinity in finite time. The fact that $g_{t}(z)$ is analytic in $z$ is clear, since the right-hand side of $(2.1)$ is analytic in $g_{t}(z)$. 
The map $g_{t}$ satisfies the so-called hydrodynamic normalization at infinity:

$$
\lim _{z \rightarrow \infty} g_{t}(z)-z=0 \text {. }
$$

Note that this uniquely determines $g_{t}$ among conformal maps from $H_{t}$ onto $\mathbb{H}$. In fact, (2.1) implies that $g_{t}$ has the power series expansion

$$
g_{t}(z)=z+\frac{2 t}{z}+\cdots, \quad z \rightarrow \infty .
$$

The fact that $g_{t} \neq g_{t^{\prime}}$ when $t^{\prime}>t$ implies that $K_{t^{\prime}} \neq K_{t}$. The relation $K_{t^{\prime}} \supset K_{t}$ is clear.

Two important properties of chordal SLE are scale-invariance and a sort of stationarity. These are summarized in the following proposition. (A similar statement appeared in [LSW01a].)

Proposition 2.1. (i) $\mathrm{SLE}_{\kappa}$ is scale-invariant in the following sense. Let $K_{t}$ be the hull of $\mathrm{SLE}_{\kappa}$, and let $\alpha>0$. Then the process $t \mapsto \alpha^{-1 / 2} K_{\alpha t}$ has the same law as $t \mapsto K_{t}$. The process $(t, z) \mapsto \alpha^{-1 / 2} g_{\alpha t}(\sqrt{\alpha} z)$ has the same law as the process $(t, z) \mapsto g_{t}(z)$.

(ii) Let $t_{0}>0$. Then the map $(t, z) \mapsto \tilde{g}_{t}(z):=g_{t+t_{0}} \circ g_{t_{0}}^{-1}\left(z+\xi\left(t_{0}\right)\right)-\xi\left(t_{0}\right)$ has the same law as $(t, z) \mapsto g_{t}(z)$; moreover, $\left(\tilde{g}_{t}\right)_{t \geq 0}$ is independent from $\left(g_{t}\right)_{0 \leq t \leq t_{0}}$.

The scaling property easily follows from the scaling property of Brownian motion, and the second property follows from the Markov property and translation invariance of Brownian motion. One just needs to write down the expression for $\partial_{t} \tilde{g}_{t}$. We leave the details as an exercise to the reader.

The following notations will be used throughout the paper.

$$
f_{t}:=g_{t}^{-1}, \quad \hat{f}_{t}(z):=f_{t}(z+\xi(t)) .
$$

The trace $\gamma$ of SLE is defined by

$$
\gamma(t):=\lim _{z \rightarrow 0} \hat{f}_{t}(z)
$$

where $z$ tends to 0 within $\mathbb{H}$. If the limit does not exist, let $\gamma(t)$ denote the set of all limit points. We say that the SLE trace is a continuous path if the limit exists for every $t$ and $\gamma(t)$ is a continuous function of $t$.

2.2. Radial SLE. Another version of $\mathrm{SLE}_{\kappa}$ is called radial $\mathrm{SLE}_{\kappa}$. It is similar to chordal SLE but appropriate for the situation where there is a distinguished point in the interior of the domain. Radial $\mathrm{SLE}_{\kappa}$ is defined as follows. Let $B(t)$ be Brownian motion on the unit circle $\partial \mathbb{U}$, started from a uniform-random point $B(0)$, and set $\xi(t):=B(\kappa t)$. The conformal maps $g_{t}$ are defined as the solution of

$$
\partial_{t} g_{t}(z)=-g_{t}(z) \frac{g_{t}(z)+\xi(t)}{g_{t}(z)-\xi(t)}, \quad g_{0}(z)=z
$$


for $z \in \mathbb{U}$. The sets $K_{t}$ and $H_{t}$ are defined as for chordal SLE. Note that the scaling property 2.1.(i) fails for radial SLE. Mainly due to this reason, chordal SLE is easier to work with. However, appropriately stated, all the main results of this paper are valid also for radial SLE. This follows from [LSW01b, Prop. 4.2], which says in a precise way that chordal and radial SLE are equivalent.

2.3. Local martingales and martingales. The purpose of this subsection is to present a slightly technical lemma giving a sufficient condition for a local martingale to be a martingale. Although we have not been able to find an appropriate reference, the lemma must be known (and is rather obvious to the experts).

See, for example, [RY99, §IV.1] for a discussion of the distinction between a local martingale and a martingale.

While the stochastic calculus needed for the rest of the paper is not much more than familiarity with Itô's formula, this subsection does assume a bit more.

Lemma 2.2. Let $B_{t}$ be stardard one dimensional Brownian motion, and let $a_{t}$ be a progressive real valued locally bounded process. Suppose that $X_{t}$ satisfies

$$
X_{t}=\int_{0}^{t} a_{s} d B_{s}
$$

and that for every $t>0$ there is a finite constant $c(t)$ such that

$$
a_{s}^{2} \leq c(t) X_{s}^{2}+c(t)
$$

for all $s \in[0, t]$ a.s. Then $X$ is a martingale.

Proof. We know that $X$ is a local martingale. Let $M>0$ be large, and let $T:=\inf \left\{t:\left|X_{t}\right| \geq M\right\}$. Then $Y_{t}:=X_{t \wedge T}$ is a martingale (where $t \wedge T=\min \{t, T\})$. Let $f(t):=\mathbf{E}\left[Y_{t}^{2}\right]$. Itô's formula then gives

$$
f\left(t^{\prime}\right)=\mathbf{E}\left[\int_{0}^{t^{\prime}} a_{s}^{2} 1_{s<T} d s\right] .
$$

Our assumption $a_{s}^{2} \leq c(t) X_{s}^{2}+c(t)$ therefore implies that for $t^{\prime} \in[0, t]$

$$
f\left(t^{\prime}\right) \leq c(t) t^{\prime}+c(t) \int_{0}^{t^{\prime}} f(s) d s .
$$

This implies $f(s)<\exp (2 c(t) s)$ for all $s \in[0, t]$, since (2.5) shows that $t^{\prime}$ cannot be the least $s \in[0, t]$ where $f(s) \geq \exp (2 c(t) s)$. Thus,

$$
\mathbf{E}\left[\langle X, X\rangle_{t \wedge T}\right]=\mathbf{E}\left[\langle Y, Y\rangle_{t}\right]=\mathbf{E}\left[Y_{t}^{2}\right]=f(t)<\exp (2 c(t) t) .
$$

Taking $M \rightarrow \infty$, we get by monotone convergence $\mathbf{E}\left[\langle X, X\rangle_{t}\right] \leq \exp (2 c(t) t)$ $<\infty$. Thus $X$ is a martingale (for example, by [RY99, IV.1.25]). 


\section{Derivative expectation}

In this section, $g_{t}$ is the $\mathrm{SLE}_{\kappa}$ flow; that is, the solution of (2.1) where $\xi(t):=B(\kappa t)$, and $B$ is standard Brownian motion on $\mathbb{R}$ starting from 0 . Our only assumption on $\kappa$ is $\kappa>0$. The goal of the section is to derive bounds on quantities related to $\mathbf{E}\left[\left|g_{t}^{\prime}(z)\right|^{a}\right]$. Another result of this nature is Lemma 6.3, which is deferred to a later section.

3.1. Basic derivative expectation. We will need estimates for the moments of $\left|\hat{f}_{t}^{\prime}\right|$. In this subsection, we will describe a change of time and obtain derivative estimates for the changed time.

For convenience, we take $B$ to be two-sided Brownian motion. The equation (2.1) can also be solved for negative $t$, and $g_{t}$ is a conformal map from $\mathbb{H}$ into a subset of $\mathbb{H}$ when $t<0$. Notice that the scale invariance (Proposition 2.1.(i)) also holds for $t<0$.

LEMMA 3.1. For all fixed $t \in \mathbb{R}$ the map $z \mapsto g_{-t}(z)$ has the same distribution as the map $z \mapsto \hat{f}_{t}(z)-\xi(t)$.

Proof. Fix $t_{1} \in \mathbb{R}$, and let

$$
\hat{\xi}_{t_{1}}(t)=\xi\left(t_{1}+t\right)-\xi\left(t_{1}\right) .
$$

Then $\hat{\xi}_{t_{1}}: \mathbb{R} \rightarrow \mathbb{R}$ has the same law as $\xi: \mathbb{R} \rightarrow \mathbb{R}$. Let

$$
\hat{g}_{t}(z):=g_{t_{1}+t} \circ g_{t_{1}}^{-1}\left(z+\xi\left(t_{1}\right)\right)-\xi\left(t_{1}\right),
$$

and note that $\hat{g}_{0}(z)=z$ and $\hat{g}_{-t_{1}}(z)=\hat{f}_{t_{1}}(z)-\xi\left(t_{1}\right)$. Since

$$
\partial_{t} \hat{g}_{t}=\frac{2}{\hat{g}_{t}+\xi\left(t_{1}\right)-\xi\left(t+t_{1}\right)}=\frac{2}{\hat{g}_{t}-\hat{\xi}_{t_{1}}(t)},
$$

the lemma follows from (2.1).

Note that (2.1) implies that $\operatorname{Im}\left(g_{t}(z)\right)$ is monotone decreasing in $t$ for every $z \in \mathbb{H}$. For $z \in \mathbb{H}$ and $u \in \mathbb{R}$ set

$$
T_{u}=T_{u}(z):=\sup \left\{t \in \mathbb{R}: \operatorname{Im}\left(g_{t}(z)\right) \geq e^{u}\right\} .
$$

We claim that for all $z \in \mathbb{H}$ a.s. $T_{u} \neq \pm \infty$. By (2.1),

$$
\left|\partial_{t} g_{t}(z)\right|=2\left|g_{t}(z)-\xi(t)\right|^{-1}
$$

Setting $\bar{\xi}(t):=\sup \{|\xi(s)|: s \in[0, t]\}$, the above implies that $\left|g_{t}(z)\right| \leq|z|+$ $\bar{\xi}(t)+2 \sqrt{t}$ for $t<\tau(z)$, since $\partial_{t}\left|g_{t}(z)\right| \leq 2 /\left(\left|g_{t}(z)\right|-\bar{\xi}(t)\right)$ whenever $\left|g_{t}(z)\right|>$ $\bar{\xi}(t)$. Setting $y_{t}:=\operatorname{Im} g_{t}(z)$, we get from $(2.1)$,

$$
-\partial_{t} \log y_{t} \geq 2(|z|+2 \bar{\xi}(t)+2 \sqrt{t})^{-2} .
$$


The law of iterated logarithms implies that the right-hand side is not integrable over $[0, \infty)$ nor over $(-\infty, 0]$. Thus, $\left|T_{u}\right|<\infty$ a.s.

We will need the formula

$$
\begin{aligned}
\partial_{t} \log \left|g_{t}^{\prime}(z)\right| & =\operatorname{Re}\left(\frac{\partial_{z} \partial_{t} g_{t}(z)}{g_{t}^{\prime}(z)}\right)=\operatorname{Re}\left(g_{t}^{\prime}(z)^{-1} \partial_{z} \frac{2}{g_{t}(z)-\xi(t)}\right) \\
& =-2 \operatorname{Re}\left(\left(g_{t}(z)-\xi(t)\right)^{-2}\right) .
\end{aligned}
$$

Set $u=u(z, t):=\log \operatorname{Im} g_{t}(z)$. Observe that (2.1) gives

$$
\partial_{t} u=-2\left|g_{t}(z)-\xi(t)\right|^{-2},
$$

and (3.3) gives

$$
\partial_{u} \log \left|g_{t}^{\prime}(z)\right|=\frac{\operatorname{Re}\left(\left(g_{t}(z)-\xi(t)\right)^{2}\right)}{\left|g_{t}(z)-\xi(t)\right|^{2}} .
$$

Fix some $\hat{z}=\hat{x}+i \hat{y} \in \mathbb{H}$. For every $u \in \mathbb{R}$, let

$$
z(u):=g_{T_{u}(\hat{z})}(\hat{z})-\xi\left(T_{u}\right), \quad \psi(u):=\frac{\hat{y}}{y(u)}\left|g_{T_{u}}^{\prime}(\hat{z})\right|,
$$

and

$$
x(u):=\operatorname{Re}(z(u)), \quad y(u):=\operatorname{Im}(z(u))=\exp (u) .
$$

TheOREm 3.2. Let $\hat{z}=\hat{x}+i \hat{y} \in \mathbb{H}$ as above. Assume that $\hat{y} \neq 1$, and set $\nu:=-\operatorname{sign}(\log \hat{y})$. Let $b \in \mathbb{R}$. Define $a$ and $\lambda$ by

$$
a:=2 b+\nu \kappa b(1-b) / 2, \quad \lambda:=4 b+\nu \kappa b(1-2 b) / 2 .
$$

Set

$$
F(\hat{z})=F_{b}(\hat{z}):=\hat{y}^{a} \mathbf{E}\left[\left(1+x(0)^{2}\right)^{b}\left|g_{T_{0}(\hat{z})}^{\prime}(\hat{z})\right|^{a}\right] .
$$

Then

$$
F(\hat{z})=\left(1+(\hat{x} / \hat{y})^{2}\right)^{b} \hat{y}^{\lambda}
$$

Before we give the short proof of the theorem, a few remarks may be of help to motivate the formulation and the proof. Our goal was to find an expression for $\mathbf{E}\left[\left|g_{T_{0}(\hat{z})}^{\prime}(\hat{z})\right|^{a}\right]$. It turns out to be more convenient to consider

$$
\bar{F}(\hat{z}):=\hat{y}^{a} \mathbf{E}\left[\left|g_{T_{0}(\hat{z})}^{\prime}(\hat{z})\right|^{a}\right]=\mathbf{E}\left[\psi(0)^{a}\right] .
$$

The obvious strategy is to find a differential equation which $\bar{F}$ must satisfy and search for a solution. The first part is not too difficult, and proceeds as follows. Let $\mathcal{F}_{u}$ denote the $\sigma$-field generated by $\left\langle\xi(t):\left(t-T_{u}\right) \nu \geq 0\right\rangle$. Note that the 
strong Markov property for $\xi$ and the chain rule imply that for $u$ between 0 and $\hat{u}:=\log \hat{y}$,

$$
\mathbf{E}\left[\psi(0)^{a} \mid \mathcal{F}_{u}\right]=\psi(u)^{a} \bar{F}(z(u)) .
$$

Hence, the right-hand side is a martingale. Observe that

$$
d x=\frac{2 x d t}{x^{2}+y^{2}}-d \xi, \quad d y=\frac{-2 y d t}{x^{2}+y^{2}}, \quad d \log \psi=\frac{4 y^{2} d t}{\left(x^{2}+y^{2}\right)^{2}} .
$$

(The latter easily follows from (3.3) and (3.4).) We assume for now that $\bar{F}$ is smooth. Itô's formula may then be calculated for the right-hand side of (3.8). Since it is a martingale, the drift term of the Itô derivative must vanish; that is,

$$
\psi^{a} \cdot \Lambda \bar{F}=0
$$

where

$$
\Lambda G:=-\frac{4 \nu a y^{2}}{\left(x^{2}+y^{2}\right)^{2}} G-\frac{2 \nu x}{x^{2}+y^{2}} \partial_{x} G+\frac{2 \nu y}{x^{2}+y^{2}} \partial_{y} G+\frac{\kappa}{2} \partial_{x}^{2} G
$$

(The $-\nu$ factor comes from the fact that $t$ is monotone decreasing with respect to the filtration $\mathcal{F}_{u}$ if and only if $\nu=1$.) Guessing a solution for the equation $\Lambda G=0$ is not too difficult (after changing to coordinates where scale invariance is more apparent). It is easy to verify that

$$
\hat{F}(x+i y)=\hat{F}_{b, \lambda}(x+i y):=\left(1+(x / y)^{2}\right)^{b} y^{\lambda},
$$

satisfies $\Lambda \hat{F}=0$. Unfortunately, $\hat{F}$ does not satisfy the boundary values $\hat{F}=1$ for $y=1$, which hold for $\bar{F}$. Consequently, the theorem gives a formula for $F$, rather than for $\bar{F}$. (Remark 3.4 concerns the problem of determining $\bar{F}$.) Assuming that $F$ is $C^{2}$, the above derivation does apply to $F$, and shows that $\Lambda F=0$. However, we have not found a clean reference to the fact that $F \in C^{2}$. Fortunately, the proof below does not need to assume this.

Proof of Theorem 3.2. Note that by (3.4)

$$
d u=-2|z|^{-2} d t \text {. }
$$

Let

$$
\hat{B}(u):=-\sqrt{2 / \kappa} \int_{t=0}^{T_{u}}|z|^{-1} d \xi
$$

Then $\hat{B}$ is a local martingale and

$$
d\langle\hat{B}\rangle=-2|z|^{-2} d t=d u .
$$

Consequently, $\hat{B}(u)$ is Brownian motion (with respect to $u$ ). Set $M_{u}:=$ $\psi(u)^{a} \hat{F}(z(u))$. Itô's formula gives

$$
d M_{u}=-2 M \frac{b x}{x^{2}+y^{2}} d \xi=\sqrt{2 \kappa} M \frac{b x}{\sqrt{x^{2}+y^{2}}} d \hat{B} .
$$


Thus $M$ is a local martingale. In fact, Lemma 2.2 then tells us that $M$ is a martingale. Consequently, we have

$$
\psi(\hat{u})^{a} \hat{F}(\hat{z})=\mathbf{E}\left[\psi(0)^{a} \hat{F}(z(0))\right]
$$

and the theorem clearly follows.

In Section 8 we will estimate the expected number of disks needed to cover the boundary of $K_{t}$. To do this, we need the following lower bound on the expectation of the derivative.

LEMMA 3.3. Let $\kappa>0$ and $b<(\kappa+4) /(4 \kappa)$, and define $a$ and $\lambda$ by (3.6) with $\nu=1$. Then there is a constant $c=c(\kappa, b)>0$ such that

$$
\mathbf{E}\left[\left|\hat{f}_{1}^{\prime}(\hat{z})\right|^{a} 1_{\operatorname{Im}\left(\hat{f}_{1}(\hat{z})\right) \geq c}\right] \geq c\left(1+(\hat{x} / \hat{y})^{2}\right)^{b} \hat{y}^{\lambda-a}
$$

holds for every $\hat{z}=\hat{x}+i \hat{y} \in \mathbb{H}$ satisfying $|\hat{z}| \leq c$.

Proof. As before, let $u:=\log y$. Set $v=v(u):=\sinh ^{-1}(x / y)$; that is, $x=y \sinh (v)$. Then Itô's formula gives

$$
d v=-\frac{d \xi}{|z|}+\frac{1}{2}(8+\kappa \nu)|z|^{-3} x d t
$$

The formula for $d v$ in terms of $\hat{B}$ and $d u$ is

$$
d v=\sqrt{\kappa / 2} d \hat{B}-\frac{x}{4|z|}(8+\kappa \nu) d u .
$$

Recall that $\psi(u)^{a} F(z(u))$ is a martingale, since $F=\hat{F}$. Define a new probability measure by

$$
\tilde{\mathbf{P}}[\mathcal{A}]:=\frac{\mathbf{E}\left[1_{\mathcal{A}}\left(1+x(0)^{2}\right)^{b} \psi(0)^{a}\right]}{\mathbf{E}\left[\left(1+x(0)^{2}\right)^{b} \psi(0)^{a}\right]},
$$

for every event $\mathcal{A}$. This is the so-called Doob-transform (or h-transform) corresponding to the martingale $\psi(u)^{a} F(z(u))$. Recall that if $\alpha(w)$ is a positive martingale for a diffusion process $d w=q_{1}(w, t) d B(t)+q_{2}(w, t) d t, t \leq t_{1}$, where $B(t)$ is Brownian motion, then for $t<t_{1}, d w=q_{1}(w, t) d \tilde{B}(t)+q_{2}(w, t) d t+$ $q_{1}(w, t) \partial_{w} \log \alpha(w) q_{1}(w, t) d t$, where $\tilde{B}$ is Brownian motion with respect to the probability measure weighted by $\alpha\left(w\left(t_{1}\right)\right)$; that is, the Doob transform of $\alpha$. This follows, for example, from Girsanov's Formula [Dur96, §2.12]. We apply this with $w=(v, \psi)$ and $u$ as the time parameter (in this case, $q_{1}$ and $q_{2}$ are vectors and $\partial_{w} \log \alpha(w)$ is a linear functional), and get by (3.12)

$$
d v=\sqrt{\kappa / 2} d \tilde{B}-\frac{x}{4|z|}(8+\kappa) d u+\frac{1}{2} \kappa \partial_{v} \log F d u,
$$


where $\tilde{B}(u)$ is Brownian motion under $\tilde{\mathbf{P}}$. This simplifies to

$$
d v=\sqrt{\kappa / 2} d \tilde{B}-\tilde{b} \tanh (v) d u,
$$

where

$$
\tilde{b}:=2-\left(b-\frac{1}{4}\right) \kappa>1 .
$$

Thus, $v(u)$ is a very simple diffusion process. When $|v|$ is large, $\tanh (v)$ is close to $\operatorname{sign}(v)$, and $v$ has almost constant drift $-\tilde{b} \operatorname{sign}(v)$, pushing it towards 0 .

At this point, do not assume $|\hat{z}| \leq c$, but only $|\hat{z}| \leq 1$. Let $\Psi:[\hat{u}, 0] \rightarrow \mathbb{R}$ be the continuous function that is equal to 1 at 0 , equal to $|\hat{u}|+1$ at $\hat{u}$, has slope $-(\tilde{b} \wedge 2+1) / 2$ in the interval $[\hat{u}, \hat{u} / 2]$ and has constant slope in the interval $[\hat{u} / 2,0]$, and let $\mathcal{A}$ be the event

$$
\mathcal{A}:=\{\forall u \in[\hat{u}, 0], \quad|v(u)| \leq \Psi(u)\} .
$$

Note that our assumption $|\hat{z}| \leq 1$ implies that $|\hat{v}| \leq|\hat{u}|+\log 2$. Since $\tilde{b}>1$ it easily follows from (3.13) that there is a constant $c_{1}=c_{1}(b, \kappa)>0$ such that $\tilde{\mathbf{P}}[\mathcal{A}]>c_{1}$. In particular, $c_{1}$ does not depend on $\hat{z}$. This means

$$
\mathbf{E}\left[1_{\mathcal{A}}\left(1+x(0)^{2}\right)^{b} \psi(0)^{a}\right] \geq c_{1} \mathbf{E}\left[\left(1+x(0)^{2}\right)^{b} \psi(0)^{a}\right]=c_{1}\left(1+(\hat{x} / \hat{y})^{2}\right)^{b} \hat{y}^{\lambda} .
$$

However, note that $v(0)$ and hence $x(0)$ are bounded on $\mathcal{A}$. Therefore, there is some constant $c_{2}>0$ such that

$$
\mathbf{E}\left[1_{\mathcal{A}}\left|g_{T_{0}}^{\prime}(\hat{z})\right|^{a}\right] \geq c_{2}\left(1+(\hat{x} / \hat{y})^{2}\right)^{b} \hat{y}^{\lambda-a} .
$$

We now estimate $T_{0}$ on the event $\mathcal{A}$. Recall that $T_{0} \leq 0$. From (3.4) we have $\partial_{u} T_{u}=-|z|^{2} / 2$ and therefore on $\mathcal{A}$

$$
\begin{aligned}
T_{0} & =-\int_{\hat{u}}^{0} \frac{y^{2}}{2} d u-\int_{\hat{u}}^{0} \frac{x^{2}}{2} d u=\frac{\hat{y}^{2}}{4}-\frac{1}{4}-\frac{1}{2} \int_{\hat{u}}^{0} \sinh (v)^{2} e^{2 u} d u \\
& \geq-\frac{1}{4}-\frac{1}{2} \int_{\hat{u}}^{0} e^{2 \Psi(u)+2 u} d u \geq-c_{3},
\end{aligned}
$$

where $c_{3}=c_{3}(b, \kappa)<\infty$ is some constant. That is, we have $T_{0} \in\left[-c_{3}, 0\right]$ on $\mathcal{A}$. On the event $T_{0} \geq-c_{3}$, we clearly have $\operatorname{Im}\left(g_{-c_{3}}(\hat{z})\right) \geq 1$ and also $\left|g_{T_{0}}^{\prime}(\hat{z})\right| \leq\left|g_{-c_{3}}^{\prime}(\hat{z})\right|$, by (3.3). Consequently, Lemma 3.1 and (3.14) give

$$
\mathbf{E}\left[\left|\hat{f}_{c_{3}}^{\prime}(\hat{z})\right|^{a} 1_{\operatorname{Im}\left(\hat{f}_{c_{3}}(\hat{z})\right) \geq 1}\right] \geq c_{4}\left(1+(\hat{x} / \hat{y})^{2}\right)^{b} \hat{y}^{\lambda-a} .
$$

This is almost the result that we need. However, we want to replace $c_{3}$ by 1 . For this, we apply scale invariance. In this procedure, the assumption $|\hat{z}| \leq 1$ needs to be replaced by $|\hat{z}| \leq 1 / \sqrt{c_{3}}$. The proof of the lemma is now complete. 
It is not too hard to see that for every constant $C>0$ the statement of the lemma can be strengthened to allow $c<|\hat{z}|<C$. The constant $c$ must then also depend on $C$.

Remark 3.4. Suppose that we take

$$
b=\frac{1}{4}+\frac{2 \nu}{\kappa},
$$

and define $a$ and $\lambda$ using (3.6). Define $\tilde{\mathbf{P}}$ as in the proof of the lemma. Then as the proof shows, $v$ becomes Brownian motion times $\sqrt{\kappa / 2}$ under $\tilde{\mathbf{P}}$, since $\tilde{b}$ vanishes. This allows a detailed understanding of the distribution of $\left|g_{T_{0}}^{\prime}(\hat{z})\right|$. For example, one can determine in this way the decay of $\mathbf{P}[\psi(0)>1 / 2]$ as $\hat{y} \searrow 0$. It also follows that for such $a, b, \lambda$, and every $A \subset \mathbb{R}$ one can write down an explicit expression for $\mathbf{E}\left[\left|g_{T_{0}}^{\prime}(\hat{z})\right|^{a} 1_{x(0) \in A}\right]$, since for every $v_{0} \in \mathbb{R}$ we have

$$
\Lambda\left(\left(1+(x / y)^{2}\right)^{b} y^{\lambda} \frac{1}{\sqrt{\nu u}} \exp \frac{\left(v_{0}-v\right)^{2}}{\nu \kappa u}\right)=0 .
$$

This equation is the PDE facet of the fact that $v$ is Brownian motion times $\sqrt{\kappa / 2}$ under $\tilde{\mathbf{P}}$. However, these results will not be needed in the present paper.

3.2. Derivative upper bounds at a fixed time $t$. From Theorem 3.2 it is not hard to obtain estimates for $\left|\hat{f}_{t}^{\prime}\right|$ :

Corollary 3.5. Let $b \in\left[0,1+4 \kappa^{-1}\right]$, and define $\lambda$ and $a$ by (3.6) with $\nu=1$. There is a constant $C(\kappa, b)$, depending only on $\kappa$ and $b$, such that the following estimate holds for all $t \in[0,1], y, \delta \in(0,1]$ and $x \in \mathbb{R}$.

$$
\mathbf{P}\left[\left|\hat{f}_{t}^{\prime}(x+i y)\right| \geq \delta y^{-1}\right] \leq C(\kappa, b)\left(1+x^{2} / y^{2}\right)^{b}(y / \delta)^{\lambda} \vartheta(\delta, a-\lambda),
$$

where

$$
\vartheta(\delta, s)= \begin{cases}\delta^{-s} & s>0 \\ 1+|\log \delta| & s=0 \\ 1 & s<0\end{cases}
$$

Proof. Note that $a \geq 0$. We assume that $\delta>y$, for otherwise the right-hand side is at least $C(\kappa, b)$, and we take $C(\kappa, b) \geq 1$. Take $z=$ $x+i y$. By Lemma 3.1, $\hat{f}_{t}^{\prime}(z)$ has the same distribution as $g_{-t}^{\prime}(z)$. Let $u_{1}:=$ $\log \operatorname{Im}\left(g_{-t}(x+i y)\right)$. Recall the notation $T_{u}$ from (3.2). Note that

$$
\left|g_{-t}^{\prime}(z) / g_{T_{u}(z)}^{\prime}(z)\right| \leq \exp \left(\left|u-u_{1}\right|\right),
$$

since $\left|\partial_{u} \log \right| g_{t}^{\prime}(z)|| \leq 1$, by (3.5). Moreover, it is clear that there is a constant $c$ such that $u_{1} \leq c$, because $t, y \leq 1$. Consequently,

$$
\mathbf{P}\left[\left|g_{-t}^{\prime}(z)\right| \geq O(1) \delta y^{-1}\right] \leq O(1) \sum_{j=\lceil\log y\rceil}^{0} \mathbf{P}\left[\left|g_{T_{j}(z)}^{\prime}(z)\right| \geq \delta y^{-1}\right]
$$


The Schwarz lemma implies that $y\left|g^{\prime}(z)\right| \leq \operatorname{Im}(g(z))$ if $g: \mathbb{H} \rightarrow \mathbb{H}$. Therefore, the above gives

$$
\mathbf{P}\left[\left|g_{-t}^{\prime}(z)\right| \geq O(1) \delta y^{-1}\right] \leq O(1) \sum_{j=\lceil\log \delta\rceil}^{0} \mathbf{P}\left[\left|g_{T_{j}(z)}^{\prime}(z)\right| \geq \delta y^{-1}\right] .
$$

By scale invariance, we have for all $j \in[\log y, 0]$

$$
\mathbf{E}\left[y^{a} e^{-j a}\left|g_{T_{j}(z)}^{\prime}(z)\right|^{a}\right] \leq F_{b}\left(e^{-j} z\right)
$$

where $F=F_{b}$ is as in Theorem 3.2. Hence

$$
\begin{aligned}
\mathbf{P}\left[\left|g_{T_{j}(z)}^{\prime}(z)\right| \geq \delta y^{-1}\right] & =\mathbf{P}\left[\left|g_{T_{j}(z)}^{\prime}(z)\right|^{a} y^{a} \delta^{-a} \geq 1\right] \\
& \leq \mathbf{E}\left[\left|g_{T_{j}(z)}^{\prime}(z)\right|^{a} y^{a} \delta^{-a}\right] \leq \delta^{-a} e^{j a} F_{b}\left(e^{-j} z\right) .
\end{aligned}
$$

Consequently, by (3.18) and Theorem 3.2,

$$
\mathbf{P}\left[\left|g_{-t}^{\prime}(z)\right| \geq O(1) \delta y^{-1}\right] \leq O(1)\left(1+x^{2} / y^{2}\right)^{b} \delta^{-a} y^{\lambda} \sum_{j=\lceil\log \delta\rceil}^{0} e^{j(a-\lambda)}
$$

The corollary follows.

3.3. Continuity of $\hat{f}_{t}(0)$. In the deterministic example of nonlocally connected hulls described in the introduction, there is a time $t_{0}$ for which $\lim _{z \rightarrow 0} \hat{f}_{t_{0}}(z)$ does not exist (the limit set is the outer boundary of the prescribed compact set). Even when the SLE trace is a continuous path, it is not always true that $(z, t) \mapsto f_{t}(z)$ extends continuously to $\overline{\mathbb{H}} \times[0, \infty)$ (this is only true for simple paths). The next theorem shows that $\hat{f}_{t}(0)=f_{t}(\xi(t))$ exists as a radial limit and is continuous. Together with the result of Section 4 below, this is enough to show that the SLE trace is a path.

Theorem 3.6. Define

$$
H(y, t):=\hat{f}_{t}(i y), \quad y>0, t \in[0, \infty) .
$$

If $\kappa \neq 8$, then a.s. $H(y, t)$ extends continuously to $[0, \infty) \times[0, \infty)$.

Proof. Fix $\kappa \neq 8$. By scale invariance, it is enough to show continuity of $H$ on $[0, \infty) \times[0,1)$. Given $j, k \in \mathbb{N}$, with $k<2^{2 j}$, let $R(j, k)$ be the rectangle

$$
R(j, k):=\left[2^{-j-1}, 2^{-j}\right] \times\left[k 2^{-2 j},(k+1) 2^{-2 j}\right],
$$

and set

$$
d(j, k):=\operatorname{diam} H(R(j, k))
$$

Take $b=(8+\kappa) /(4 \kappa)$ and let $a$ and $\lambda$ be given by (3.6) with $\nu=1$. Note that $\lambda>2$. Set $\sigma_{0}:=(\lambda-2) / \max \{a, \lambda\}$, and let $\sigma \in\left(0, \sigma_{0}\right)$. Our objective is to 
prove

$$
\sum_{j=0}^{\infty} \sum_{k=0}^{2^{2 j}-1} \mathbf{P}\left[d(j, k) \geq 2^{-j \sigma}\right]<\infty .
$$

Fix some such pair $(j, k)$. The idea of the proof is to decompose the time interval $\left[k 2^{-2 j},(k+1) 2^{-2 j}\right]$ into (random) sub-intervals using partition points $\hat{t}_{N}, \hat{t}_{N-1}, \ldots, \hat{t}_{0}$ such that the change of $\xi$ on each interval is controlled. Then it is not hard to control $\hat{f}_{t}$ on each sub-interval. Now, the details. Set $t_{0}=(k+1) 2^{-2 j}$. Inductively, let

$$
t_{n+1}:=\sup \left\{t<t_{n}:\left|\xi(t)-\xi\left(t_{n}\right)\right|=2^{-j}\right\},
$$

and let $N$ be the least $n \in \mathbb{N}$ such that $t_{N} \leq t_{0}-2^{-2 j}$. Also set $t_{\infty}:=$ $t_{0}-2^{-2 j}=k 2^{-2 j}$ and

$$
\hat{t}_{n}:=\max \left\{t_{n}, t_{\infty}\right\} .
$$

Observe that the scaling property of Brownian motion shows that there is a constant $\rho<1$, which does not depend on $j$ or $k$, such that $\mathbf{P}[N>1]=\rho$. Moreover, the Markov property implies that $\mathbf{P}[N \geq m+1 \mid N \geq m] \leq \rho$ for every $m \in \mathbb{N}$. In particular, $\mathbf{P}[N>m] \leq \rho^{m}$.

For every $s \in[0, \infty)$, the map $\hat{f}_{s}$ is measurable with respect to the $\sigma$-field generated by $\xi(t)$ for $t \in[0, s]$, while $\hat{t}_{n}$ is determined by $\xi(t)$ for $t \geq t_{n}$ (i.e., $\hat{t}_{n}$ is a stopping time for the reversed time filtration). Therefore, by the strong Markov property, for every $n \in \mathbb{N}, s \in\left[t_{\infty}, t_{0}\right]$ and $\delta>0$,

$$
\mathbf{P}\left[\left|\hat{f}_{\hat{t}_{n}}^{\prime}\left(i 2^{-j}\right)\right|>\delta \mid \hat{t}_{n}=s\right]=\mathbf{P}\left[\left|\hat{f}_{s}^{\prime}\left(i 2^{-j}\right)\right|>\delta\right],
$$

and consequently,

$$
\mathbf{P}\left[\left|\hat{f}_{\hat{t}_{n}}^{\prime}\left(i 2^{-j}\right)\right|>\delta \mid \hat{t}_{n}>t_{\infty}\right] \leq \sup _{s \in[0,1]} \mathbf{P}\left[\left|\hat{f}_{s}^{\prime}\left(i 2^{-j}\right)\right|>\delta\right] .
$$

This allows us to estimate,

$$
\begin{aligned}
\mathbf{P}[\exists n & \left.\in \mathbb{N}:\left|\hat{f}_{\hat{t}_{n}}^{\prime}\left(i 2^{-j}\right)\right|>\delta\right] \\
& \leq \mathbf{P}\left[\left|\hat{f}_{t_{\infty}}^{\prime}\left(i 2^{-j}\right)\right|>\delta\right]+\sum_{n=0}^{\infty} \mathbf{P}\left[\hat{t}_{n}>t_{\infty}\right] \mathbf{P}\left[\left|\hat{f}_{\hat{t}_{n}}^{\prime}\left(i 2^{-j}\right)\right|>\delta \mid \hat{t}_{n}>t_{\infty}\right] \\
& \leq \mathbf{E}[N+1] \sup _{s \in[0,1]} \mathbf{P}\left[\left|\hat{f}_{s}^{\prime}\left(i 2^{-j}\right)\right|>\delta\right] \leq O(1) \sup _{s \in[0,1]} \mathbf{P}\left[\left|\hat{f}_{s}^{\prime}\left(i 2^{-j}\right)\right|>\delta\right] .
\end{aligned}
$$

By Corollary 3.5, and because $\sigma<(\lambda-2) / \max \{a, \lambda\}$, this gives

$$
\mathbf{P}\left[\exists n \in \mathbb{N}:\left|\hat{f}_{\hat{t}_{n}}^{\prime}\left(i 2^{-j}\right)\right|>2^{j} 2^{-j \sigma} / j^{2}\right] \leq O(1) 2^{-2 j} 2^{-\varepsilon j},
$$

for some $\varepsilon=\varepsilon(\kappa)>0$. Let $S$ be the rectangle

$$
S:=\left\{x+i y:|x| \leq 2^{-j+3}, y \in\left[2^{-j-1}, 2^{-j+3}\right]\right\} .
$$


We claim that

$$
H(R(j, k)) \subset \bigcup_{n=0}^{N} \hat{f}_{\hat{t}_{n}}(S)
$$

and

$$
\forall n \in \mathbb{N} \quad \hat{f}_{\hat{t}_{n}}(S) \cap \hat{f}_{\hat{t}_{n+1}}(S) \neq \emptyset .
$$

Indeed, to prove $(3.21)$, let $t \in\left[\hat{t}_{n+1}, \hat{t}_{n}\right]$ and $y \in\left[2^{-j-1}, 2^{-j}\right]$. Then we may write

$$
\hat{f}_{t}(i y)=\hat{f}_{\hat{t}_{n+1}}\left(g_{\hat{t}_{n+1}}\left(\hat{f}_{t}(i y)\right)-\xi\left(\hat{t}_{n+1}\right)\right)
$$

We want to prove $(3.21)$ by showing that $g_{\hat{t}_{n+1}}\left(\hat{f}_{t}(i y)\right)-\xi\left(\hat{t}_{n+1}\right) \in S$. Set $\varphi(s):=g_{s}\left(\hat{f}_{t}(i y)\right)$ for $s \leq t$. Then $\varphi(t)=i y+\xi(t)$ and by $(2.1)$

$$
\partial_{s} \varphi(s)=2(\varphi(s)-\xi(s))^{-1} \text {. }
$$

Note that $\partial_{s} \operatorname{Im}(\varphi(s))<0$, and hence $\operatorname{Im}(\varphi(s)) \geq \operatorname{Im}(\varphi(t)) \geq 2^{-j-1}$. This then implies that $\left|\partial_{s} \varphi(s)\right| \leq 2^{j+2}$, and therefore $\left|t-\hat{t}_{n+1}\right| \leq 2^{-2 j}$ gives $\left|\varphi\left(\hat{t}_{n+1}\right)-\varphi(t)\right| \leq 2^{2-j}$. Since $\left|\xi(t)-\xi\left(\hat{t}_{n+1}\right)\right| \leq 2^{1-j}$, by (3.23) this gives $\hat{f}_{t}(i y) \in \hat{f}_{\hat{t}_{n+1}}(S)$ and verifies (3.21). We also have (3.22), because taking $t=\hat{t}_{n}$ in the above gives $\hat{f}_{\hat{t}_{n}}(i y) \in \hat{f}_{\hat{t}_{n+1}}(S)$. Since $\left|\hat{f}_{t}^{\prime}(z)\right| /\left|\hat{f}_{t}^{\prime}\left(i 2^{-j}\right)\right|$ is bounded by some constant if $z \in S$ (this follows from the Koebe distortion theorem, see $[$ Pom92, $§ 1.3])$, we find that

$$
\operatorname{diam}\left(\hat{f}_{t}(S)\right) \leq O(1) 2^{-j}\left|\hat{f}_{t}^{\prime}\left(i 2^{-j}\right)\right|
$$

Therefore, the relations (3.21) and (3.22) give

$$
\begin{aligned}
d(j, k) & \leq \sum_{n=0}^{N} \operatorname{diam}\left(\hat{f}_{\hat{t}_{n}}(S)\right) \leq O(1) 2^{-j} \sum_{n=0}^{N}\left|\hat{f}_{\hat{t}_{n}}^{\prime}\left(i 2^{-j}\right)\right| \\
& \leq O(1) 2^{-j} N \max \left\{\left|\hat{f}_{\hat{t}_{n}}^{\prime}\left(i 2^{-j}\right)\right|: n=0,1, \ldots, N\right\} .
\end{aligned}
$$

By (3.20), we get

$$
\begin{aligned}
\mathbf{P}\left[d(j, k)>2^{-j \sigma}\right] & \leq \mathbf{P}\left[N>j^{2}\right]+O(1) 2^{-2 j} 2^{-\varepsilon j} \\
& \leq \rho^{j^{2}}+O(1) 2^{-2 j} 2^{-\varepsilon j} \leq O(1) 2^{-2 j} 2^{-\varepsilon j}
\end{aligned}
$$

which proves (3.19).

From (3.19) we conclude that a.s. there are at most finitely many pairs $j, k \in \mathbb{N}$ with $k \leq 2^{2 j}-1$ such that $d(j, k)>2^{-j \sigma}$. Hence $d(j, k) \leq C(\omega) 2^{-j \sigma}$ for all $j, k$, where $C=C(\omega)$ is random (and the notation $C(\omega)$ is meant to suggest that). Let $\left(y^{\prime}, t^{\prime}\right)$ and $\left(y^{\prime \prime}, t^{\prime \prime}\right)$ be points in $(0,1)^{2}$. Let $j_{1}$ be the smallest integer larger than $\min \left\{-\log _{2} y^{\prime},-\log _{2} y^{\prime \prime},-\frac{1}{2} \log _{2}\left|t^{\prime}-t^{\prime \prime}\right|\right\}$. Note that a rectangle $R\left(j_{1}, k^{\prime}\right)$ that intersects the line $t=t^{\prime}$ is adjacent to a rectangle 
$R\left(j_{1}, k^{\prime \prime}\right)$ that intersects the line $t=t^{\prime \prime}$. Consequently, $\left|H\left(y^{\prime}, t^{\prime}\right)-H\left(y^{\prime \prime}, t^{\prime \prime}\right)\right| \leq$ $\sum_{j \geq j_{1}}\left(d\left(j, k_{j}^{\prime}\right)+d\left(j, k_{j}^{\prime \prime}\right)\right) \leq O(1) C(\omega) 2^{-\sigma j_{1}}$, where $R\left(j, k_{j}^{\prime}\right)$ is a rectangle meeting the line $t=t^{\prime}$ and $R\left(j, k_{j}^{\prime \prime}\right)$ is a rectangle meeting the line $t=t^{\prime \prime}$. This shows that for every $t_{0} \in[0,1)$ the limit of $H(y, t)$ as $(y, t) \rightarrow\left(0, t_{0}\right)$ exists, and thereby extends the definition of $H$ to $[0, \infty) \times[0,1)$. Since $H$ is clearly continuous in $(0, \infty) \times[0, t)$, the proof is now complete.

For $\kappa=8$, we are unable to prove Theorem 3.6. However, a weaker result does follow easily, namely, a.s. for almost every $t \geq 0$ the $\operatorname{limit} \lim _{y \backslash 0} \hat{f}_{t}(i y)$ exists.

Update. It follows from [LSW] and the results of the current paper that the Theorem holds also when $\kappa=8$.

\section{Reduction}

The following theorem provides a criterion for hulls to be generated by a continuous path. In this section we do not assume that $\xi$ is a (time scaled) Brownian motion.

ThEOREM 4.1. Let $\xi:[0, \infty) \rightarrow \mathbb{R}$ be continuous, and let $g_{t}$ be the corresponding solution of $(2.1)$. Assume that $\beta(t):=\lim _{y \backslash 0} g_{t}^{-1}(\xi(t)+i y)$ exists for all $t \in[0, \infty)$ and is continuous. Then $g_{t}^{-1}$ extends continuously to $\overline{\mathbb{H}}$ and $H_{t}$ is the unbounded connected component of $\mathbb{H} \backslash \beta([0, t])$, for every $t \in[0, \infty)$.

In the proof, the following basic properties of conformal maps will be needed. Suppose that $g: D \rightarrow \mathbb{U}$ is a conformal homeomorphism. If $\alpha$ : $[0,1) \rightarrow D$ is a path such that the limit $l_{1}=\lim _{t / 1} \alpha(t)$ exists, then $l_{2}=$ $\lim _{t / 1} g \circ \alpha$ exists, too. (However, if $\alpha:[0,1) \rightarrow \mathbb{U}$ is a path such that

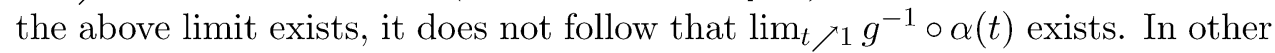
words, it is essential that the image of $g$ is a nice domain such as $\mathbb{U}$.) Moreover, $\lim _{t / 1} g^{-1}\left(t l_{2}\right)$ exists and equals $l_{1}$. Consequently, if $\tilde{\alpha}:[0,1) \rightarrow D$ is another path with $\lim _{t / 1} \tilde{\alpha}$ existing and with $\lim _{t / 1} g \circ \alpha(t)=\lim _{t \nearrow_{1}} g \circ \tilde{\alpha}(t)$, then $\lim _{t \nearrow_{1}} \alpha(t)=\lim _{t \nearrow 1} \tilde{\alpha}(t)$.

These statements are well known and easily established, for example with the notion of extremal length. See [Pom92, Prop. 2.14] for the first statement and [Ahl73, Th. 3.5] implies the second claim.

Proof. Let $S(t) \subset \overline{\mathbb{H}}$ be the set of limit points of $g_{t}^{-1}(z)$ as $z \rightarrow \xi(t)$ in $\mathbb{H}$. Fix $t_{0} \geq 0$, and let $z_{0} \in S\left(t_{0}\right)$. We want to show that $z_{0} \in \overline{\beta\left(\left[0, t_{0}\right)\right)}$, and hence $z_{0} \in \beta\left(\left[0, t_{0}\right]\right)$. Fix some $\varepsilon>0$. Let

$$
t^{\prime}:=\sup \left\{t \in\left[0, t_{0}\right]: K_{t} \cap \overline{D\left(z_{0}, \varepsilon\right)}=\emptyset\right\}
$$


where $D\left(z_{0}, \varepsilon\right)$ is the open disk of radius $\varepsilon$ about $z_{0}$. We first show that

$$
\beta\left(t^{\prime}\right) \in \overline{D\left(z_{0}, \varepsilon\right)} \text {. }
$$

Indeed, $D\left(z_{0}, \varepsilon\right) \cap H_{t_{0}} \neq \emptyset$ since $z_{0} \in S\left(t_{0}\right)$. Let $p \in D\left(z_{0}, \varepsilon\right) \cap H_{t_{0}}$, and let $p^{\prime} \in K_{t^{\prime}} \cap \overline{D\left(z_{0}, \varepsilon\right)}$. Let $p^{\prime \prime}$ be the first point on the line segment from $p$ to $p^{\prime}$ which is in $K_{t^{\prime}}$. We want to show that $\beta\left(t^{\prime}\right)=p^{\prime \prime}$. Let $L$ be the line segment $\left[p, p^{\prime \prime}\right)$, and note that $L \subset H_{t^{\prime}}$. Hence $g_{t^{\prime}}(L)$ is a curve in $\mathbb{H}$ terminating at a point $x \in \mathbb{R}$. If $x \neq \xi\left(t^{\prime}\right)$, then $g_{t}(L)$ terminates at points $x(t) \neq \xi(t)$ for all $t<t^{\prime}$ sufficiently close to $t^{\prime}$. Because $g_{\tau}\left(p^{\prime \prime}\right)$ has to hit the singularity $\xi(\tau)$ at some time $\tau \leq t^{\prime}$, this implies $p^{\prime \prime} \in K_{t}$ for $t<t^{\prime}$ close to $t^{\prime}$. This contradicts the definition of $t^{\prime}$ and shows $x=\xi\left(t^{\prime}\right)$. Now $\beta\left(t^{\prime}\right)=p^{\prime \prime}$ follows because the conformal map $g_{t^{\prime}}^{-1}$ of $\mathbb{H}$ cannot have two different limits along two arcs with the same terminal point.

Having established (4.1), since $\varepsilon>0$ was arbitrary, we conclude that $z_{0} \in \overline{\beta\left(\left[0, t_{0}\right)\right)}$ and hence $z_{0} \in \beta\left(\left[0, t_{0}\right]\right)$. This gives $S(t) \subset \beta([0, t])$ for all $t \geq 0$. We now show that $H_{t}$ is the unbounded component of $\mathbb{H} \backslash \overline{\bigcup_{\tau \leq t} S(\tau)}$. First, $H_{t}$ is connected and disjoint from $\overline{\bigcup_{\tau \leq t} S(\tau)}$. On the other hand, as the argument in the previous paragraph shows, $\partial H_{t} \cap \mathbb{H}$ is contained in $\overline{\bigcup_{\tau \leq t} S(\tau)}$. Therefore, $H_{t}$ is a connected component of $\mathbb{H} \backslash \overline{\bigcup_{\tau \leq t} S(\tau)}$; that is, $H_{t}$ is the unbounded connected component of $\mathbb{H} \backslash \beta([0, t])$. Since $\beta$ is a continuous path, it follows from [Pom92, Th. 2.1] that $g_{t}^{-1}$ extends continuously to $\overline{\mathbb{H}}$ (which also proves that $S(t)=\{\beta(t)\})$.

\section{Continuity}

We have now established all the results needed to show that the $\mathrm{SLE}_{\kappa}$ trace is a continuous path a.s.

THEOREM 5.1 (Continuity). Let $\kappa \neq 8$. The following statements hold almost surely. For every $t \geq 0$ the limit

$$
\gamma(t):=\lim _{z \rightarrow 0, z \in \mathbb{H}} \hat{f}_{t}(z)
$$

exists, $\gamma:[0, \infty) \rightarrow \overline{\mathbb{H}}$ is a continuous path, and $H_{t}$ is the unbounded component of $\mathbb{H} \backslash \gamma([0, t])$.

We believe the theorem to be valid also for $\kappa=8$. (This is stated as Conjecture 9.1.) Despite repeated efforts, the proof eluded us.

Update. This extension to $\kappa=8$ is proved in [LSW].

Proof of Theorem 5.1. By Theorem 3.6, a.s. $\lim _{y \searrow_{0}} \hat{f}_{t}(i y)$ exists for all $t$ and is continuous. Therefore we can apply Theorem 4.1, and the theorem follows. 
It follows from Theorem 5.1 that $f_{t}$ extends continuously to $\overline{\mathbb{H}}$ a.s. The next result gives more information about the regularity of $f_{t}$ on $\overline{\mathbb{H}}$. It neither follows from Theorem 5.1, nor does it imply 5.1.

THEOREM 5.2 (Hölder continuity). For every $\kappa \neq 4$ there is some $h(\kappa)>0$ such that for every bounded set $A \subset \mathbb{H}$ and every $t>0$, a.s. $\hat{f}_{t}$ is Hölder continuous with exponent $h(\kappa)$ on $A$,

$$
\left|\hat{f}_{t}(z)-\hat{f}_{t}\left(z^{\prime}\right)\right| \leq C\left|z-z^{\prime}\right|^{h(\kappa)} \quad \text { for all } \quad z, z^{\prime} \in A
$$

where $C=C(\omega, t, A)$ is random and may depend on $t$ and $A$. Moreover, $\lim _{\kappa \searrow 0} h(\kappa)=\frac{1}{2}$ and $\lim _{\kappa / \infty} h(\kappa)=1$.

Since $f_{t}(z)-z \rightarrow 0$ as $z \rightarrow \infty$, it easily follows that for every $t$ a.s.

$$
\left|\hat{f}_{t}(z)-\hat{f}_{t}\left(z^{\prime}\right)\right| \leq C(\omega, t) \max \left(\left|z-z^{\prime}\right|,\left|z-z^{\prime}\right|^{h(\kappa)}\right) .
$$

We do not believe that the theorem holds for $\kappa=4$, for then the trace is a simple path which "almost" touches itself.

Update. For $\kappa \leq 4$, the fact that $\gamma$ is a simple path in $\mathbb{H}$, Theorem 6.1 below, implies $h(\kappa) \leq 1 / 2$. Thus the estimate $\lim _{\kappa \backslash 0} h(\kappa)=\frac{1}{2}$ is best possible. On the other hand, this nonsmoothness of $\hat{f}_{t}$ is localized at $\hat{f}_{t}^{-1}(0)$ : Joan Lind (manuscript in preparation) has shown that the Hölder exponent $h(\kappa)$ of $\left(\hat{f}_{t}(\sqrt{z})\right)^{2}$ satisfies $\lim _{\kappa \backslash 0} h(\kappa)=1$.

Proof. Fix $\kappa \neq 4$ and $t>0$. By scaling, we may assume $0<t \leq 1$ and $A=[-1,1] \times(0,1]$. Denote

$$
z_{j, n}=(j+i) 2^{-n}, \quad 0 \leq n<\infty, \quad-2^{n}<j<2^{n} .
$$

We will first show that there is an $h=h(\kappa)>0$ such that a.s.

$$
\left|\hat{f}_{t}^{\prime}\left(z_{j, n}\right)\right| \leq C(\omega, t) 2^{n(1-h)}, \quad \forall j, n .
$$

Using Corollary 3.5 with $\delta=2^{-n h}$ we have

$$
\mathbf{P}\left[\left|\hat{f}_{t}^{\prime}\left(z_{j, n}\right)\right| \geq 2^{n(1-h)}\right] \leq C(\kappa, b)\left(1+2^{2 n}\right)^{b} 2^{-n(1-h) \lambda} \vartheta\left(2^{-n h}, a-\lambda\right) .
$$

Hence

$$
\sum_{n=0}^{\infty} \sum_{j=-2^{n}}^{2^{n}} \mathbf{P}\left[\left|\hat{f}_{t}^{\prime}\left(z_{j, n}\right)\right| \geq 2^{n(1-h)}\right]<\infty
$$

provided that

$$
1+2 b-(1-h) \lambda<0 \text { and } a-\lambda \leq 0
$$

or that

$$
1+2 b-\lambda+a h<0 \text { and } a-\lambda \geq 0
$$


If $0<\kappa \leq 12, b=1 / 4+1 / \kappa$ and $h<(\kappa-4)^{2} /((\kappa+4)(\kappa+12))$, the first condition is satisfied. For $\kappa>12, b=4 / \kappa$ and $h<1 / 2-4 / \kappa$ the second condition is satisfied, and (5.2) follows.

To see that one can actually achieve $h(\kappa) \rightarrow 1 / 2$ as $\kappa \searrow 0$, set $b:=-1 / 2+$ $\sqrt{1 / 2+2 / \kappa}$ for $0<\kappa<4$, and let $h$ be smaller than but close to $(\lambda-1-2 b) / \lambda$. To get $\lim _{\kappa / \infty} h(\kappa)=1$, take $b:=\left(\sqrt{2 \kappa} \sqrt{\kappa^{2}+10 \kappa+16}-2 \kappa\right) /\left(\kappa^{2}+4 \kappa\right)$ for $\kappa \geq 2(3+\sqrt{17})$ and let $h$ be smaller than but close to $(\lambda-1-2 b) / a$.

From the Koebe distortion Theorem and (5.2) we obtain

$$
\left|\hat{f}_{t}^{\prime}(z)\right| \leq O(1) C(\omega, t) y^{h-1}
$$

for all $z \in A$. It is well-known and easy to see, by integrating $\left|f^{\prime}\right|$ over the hyperbolic geodesic from $z$ to $z^{\prime}$ (similarly to the end of the proof of Theorem 3.6), that this implies Hölder continuity with exponent $h$ on $A$. The theorem follows.

The following corollary is an immediate consequence of Theorem 5.2. In Section 8 below, we will present more precise estimates.

Corollary 5.3. For $\kappa \neq 4$ and every $t$, the Hausdorff dimension of $\partial K_{t}$ a.s. satisfies

$$
\operatorname{dim} \partial K_{t}<2
$$

In particular, a.s. area $\partial K_{t}=0$.

Proof. By [JM95] (see also [KR97] for an easier proof), the boundary of the image of a disk under a Hölder continuous conformal map has Hausdorff dimension bounded away from 2. Consider the conformal map $T(z)=(z-i) /(z+i)$ from $\mathbb{H}$ onto $\mathbb{U}$. By (5.1), $T \circ f_{t} \circ T^{-1}$ is a.s. Hölder continuous in $\mathbb{U}$. Since $T$ preserves Hausdorff dimension, the corollary follows.

\section{Phases}

In this section, we will investigate the topological behavior of SLE, and will identify three very different phases for the parameter $\kappa$, namely, $[0,4]$, $(4,8)$, and $[8, \infty)$.

The following result was conjectured in [Sch00]. There, it was proved that for $\kappa>4$, a.s. $K_{t}$ is not a simple path. The proof was based on the calculation of the harmonic measure $F(x)$ below, which we will repeat here for the convenience of the reader.

THEOREM 6.1. In the range $\kappa \in[0,4]$, the $\mathrm{SLE}_{\kappa}$ trace $\gamma$ is a.s. a simple path and $\gamma[0, \infty) \subset \mathbb{H} \cup\{0\}$. 
Lemma 6.2. Let $\kappa \in[0,4]$, and let $\gamma$ be the $\mathrm{SLE}_{\kappa}$ trace. Then a.s.

$$
\gamma[0, \infty) \subset \mathbb{H} \cup\{0\} .
$$

Set $Y_{x}(t):=g_{t}(x)-\xi(t), x \in \mathbb{R}, t \geq 0$. Then $Y_{x}(t) / \sqrt{\kappa}$ is a Bessel process of dimension $1+4 / \kappa$. The basic theory of Bessel processes then tells us that a.s. $Y_{x}(t) \neq 0$ for $t \geq 0, x \neq 0$ and $\kappa \in[0,4]$, which amounts to $x \neq K_{t}$. However, we will prove this for the convenience of the reader unfamiliar with Bessel processes.

Proof. Let $b>a>0$. For $x \in[a, b]$, let

$$
T=T_{x}:=\inf \left\{t \geq 0: Y_{x}(t) \notin(a, b)\right\} .
$$

Set

$$
\hat{F}(x):= \begin{cases}x^{(\kappa-4) / \kappa} & \kappa \neq 4 \\ \log (x) & \kappa=4\end{cases}
$$

and

$$
F(x):=\frac{\hat{F}(x)-\hat{F}(a)}{\hat{F}(b)-\hat{F}(a)} .
$$

Itô's formula shows that $F\left(Y_{x}(t \wedge T)\right)$ is a local martingale, and since $F$ is bounded in $[a, b]$, this is a martingale. Consequently, the optional sampling theorem gives $F(x)=\mathbf{E}\left[F\left(Y_{x}(T)\right]=\mathbf{P}\left[Y_{x}(T)=b\right]\right.$.

Note that $F(x) \rightarrow 1$ when $a \searrow 0$. (That's where the assumption $\kappa \leq 4$ is crucial.) Hence, given $x>0$, a.s. for all $b>x$, there is some $s>0$ such that $g_{t}(x)$ is well defined for $t \in[0, s], \inf \left\{Y_{x}(t): t \in[0, s]\right\}>0$ and $Y_{x}(s)=b$. Note that the Itô derivative of $Y_{x}(t)$ (with respect to $t$ ) is

$$
d Y_{x}=\left(2 / Y_{x}\right) d t+d \xi .
$$

It follows easily that a.s. $Y_{x}(t)$ does not escape to $\infty$ in finite time. Observe that if $x^{\prime}>x$, then $Y_{x^{\prime}}(t) \geq Y_{x}(t)$. Therefore, a.s. for every $x>0$ we have $Y_{x}(t)$ well defined and in $(0, \infty)$ for all $t \geq 0$. This implies that a.s. for every $x>0$ and every $s>0$ there is some neighborhood $N$ of $x$ in $\mathbb{C}$ such that the differential equation (2.1) has a solution in the range $z \in N, t \in[0, s]$. This proves that a.s. $\gamma[0, \infty)$ does not intersect $(0, \infty)$. The proof that it a.s. does not intersect $(-\infty, 0)$ is the same.

Proof of Theorem 6.1. Let $t_{2}>t_{1}>0$. The theorem will be established by proving that $\gamma\left[0, t_{1}\right] \cap \gamma\left[t_{2}, \infty\right)=\emptyset$. Let $s \in\left(t_{1}, t_{2}\right)$ be rational, and set

$$
\hat{g}_{t}(z):=g_{t+s} \circ g_{s}^{-1}(z+\xi(s))-\xi(s) .
$$

By Proposition $2.1\left(\hat{g}_{t}: t \geq 0\right)$ has the same distribution as $\left(g_{t}: t \geq 0\right)$. Let $\hat{\gamma}_{s}(t)$ be the trace for the collection $\left(\hat{g}_{t}: t \geq 0\right)$; that is,

$$
\hat{\gamma}_{s}(t)=g_{s} \circ g_{t+s}^{-1}(\xi(t+s))-\xi(s)=g_{s} \circ \gamma(t+s)-\xi(s) .
$$


We know from Lemma 6.2 that a.s. for all rational $s \in\left[t_{1}, t_{2}\right]$,

$$
\hat{\gamma}_{s}[0, \infty) \subset \mathbb{H} \cup\{0\}
$$

Consequently, by applying $g_{s}^{-1}$, we conclude that a.s. for every rational $s>0$,

$$
\gamma[s, \infty) \cap\left(\mathbb{R} \cup K_{s}\right)=\{\gamma(s)\} .
$$

Since $g_{t_{2}} \circ g_{t_{1}}^{-1}$ is not the identity, it follows from Theorem 5.1 that there is some $s \in\left(t_{1}, t_{2}\right)$ such that $\gamma(s) \notin \mathbb{R} \cup K_{t_{1}}$, and this holds for an open set of $s \in\left(t_{1}, t_{2}\right)$. In particular, there is some rational $s \in\left(t_{1}, t_{2}\right)$ with $\gamma(s) \notin \mathbb{R} \cup K_{t_{1}}$. Since $\gamma\left[0, t_{1}\right] \subset K_{t_{1}} \cup \mathbb{R}$, it follows that $\gamma\left[0, t_{1}\right] \cap \gamma\left[t_{2}, \infty\right)=\emptyset$, proving the theorem.

Recall the definition of the hypergeometric function in $|z|<1$,

$$
{ }_{2} F_{1}\left(\eta_{0}, \eta_{1}, \eta_{2}, z\right):=\sum_{n=0}^{\infty} \frac{\left(\eta_{0}\right)_{n}\left(\eta_{1}\right)_{n}}{\left(\eta_{2}\right)_{n} n !} z^{n}
$$

where $(\eta)_{n}:=\prod_{j=0}^{n-1}(\eta+j)$. Let

$$
\hat{G}(x+i y)=\hat{G}_{a, \kappa}(x+i y):={ }_{2} F_{1}\left(\eta_{0}, \eta_{1}, 1 / 2, x^{2} /\left(x^{2}+y^{2}\right)\right),
$$

where

$$
\eta_{j}=\eta_{j}(a, \kappa):=\frac{1}{2}-\frac{2}{\kappa}-(-1)^{j} \frac{\sqrt{32 a \kappa+(2 \kappa-8)^{2}}}{4 \kappa}
$$

Lemma 6.3. Let $\kappa>0$ and $z=x+i y \in \mathbb{H}$. Then the limit

$$
Z(z):=\lim _{t \nearrow \tau(z)} y\left|g_{t}^{\prime}(z)\right| / \operatorname{Im}\left(g_{t}(z)\right)
$$

exists a.s. We have $Z(z)=\infty$ a.s. if $\kappa \geq 8$ and $Z(z)<\infty$ a.s. if $\kappa<8$. Moreover, for all $a \in \mathbb{R}$ we have

$$
\mathbf{E}\left[Z(z)^{a}\right]= \begin{cases}\hat{G}_{a, \kappa}(z) / \hat{G}_{a, \kappa}(1) & a<1-\kappa / 8, \kappa<8 \\ \infty & a \geq 1-\kappa / 8, \quad a>0 \\ 0 & a<0, \kappa \geq 8\end{cases}
$$

Before proving the lemma, let us discuss its geometric meaning. For $z_{0} \in \mathbb{H}$ and $t<\tau\left(z_{0}\right)$ we have $z_{0} \in H_{t}$. Set $r_{t}:=\operatorname{dist}\left(z_{0}, \partial H_{t}\right)$, and let $\phi: \mathbb{H} \rightarrow \mathbb{U}$ be a conformal homeomorphism satisfying $\phi\left(g_{t}\left(z_{0}\right)\right)=0$. Note that $\left|\phi^{\prime}\left(g_{t}\left(z_{0}\right)\right)\right|=\left(2 \operatorname{Im} g_{t}\left(z_{0}\right)\right)^{-1}$. Hence, the Schwarz lemma applied to the map $z \mapsto \phi \circ g_{t}\left(z_{0}+r_{t} z\right)$ proves $r_{t}\left|g_{t}^{\prime}\left(z_{0}\right)\right| \leq 2 \operatorname{Im} g_{t}\left(z_{0}\right)$. On the other hand, the Koebe $1 / 4$ Theorem applied to the function $g_{t}^{-1} \circ \phi^{-1}$ implies that $\operatorname{Im} g_{t}\left(z_{0}\right) \leq 2 r_{t}\left|g_{t}^{\prime}\left(z_{0}\right)\right|$. Therefore, since $\lim _{t / \tau\left(z_{0}\right)} r_{t}=\operatorname{dist}\left(z_{0}, \gamma[0, \infty) \cup \mathbb{R}\right)$,

$$
Z\left(z_{0}\right)^{-1} \operatorname{Im} z_{0} / 2 \leq \operatorname{dist}\left(z_{0}, \gamma[0, \infty) \cup \mathbb{R}\right) \leq 2 Z\left(z_{0}\right)^{-1} \operatorname{Im} z_{0} .
$$


In particular, the lemma tells us that $\gamma[0, \infty)$ is dense in $\mathbb{H}$ if and only if $\kappa \geq 8$. (For $\kappa=8$ we have not proven that $\gamma$ is a path. In that case, $\gamma[0, \infty)$ is defined as the union over $t$ of all limit points of sequences $\hat{f}_{t}\left(z_{j}\right)$ where $z_{j} \rightarrow 0$ in $\mathbb{H}$. By an argument in the proof of Theorem 4.1, $\overline{\bigcup_{t \geq 0} \partial K_{t}}=\overline{\gamma[0, \infty)}$.)

The way one finds the function $\hat{G}$ is explained within the proof below, and is similar to the situation in Theorem 3.2.

Proof. Let $G(z)=G_{a, \kappa}(z):=\mathbf{E}\left[Z(z)^{a}\right]$. Fix some $\hat{z}=\hat{x}+i \hat{y} \in \mathbb{H}$, and abbreviate $Z:=Z(\hat{z})$. Let $z_{t}=x_{t}+i y_{t}:=g_{t}(\hat{z})-\xi(t)$. From (3.9) we find that

$$
\partial_{t} \log \left|g_{t}^{\prime}(z) / y_{t}\right|=4 y_{t}^{2}\left|z_{t}\right|^{-4}
$$

which implies that

$$
Z=\exp \left(\int_{0}^{\tau(\hat{z})} 4 y_{t}^{2}\left|z_{t}\right|^{-4} d t\right)=\exp \left(\int_{0}^{\tau(\hat{z})} \frac{-2 y_{t}^{2}}{x_{t}^{2}+y_{t}^{2}} d \log y_{t}\right) .
$$

In particular, the limit in the definition of $Z$ exists a.s. and $G(\hat{z})=\mathbf{E}\left[Z^{a}\right]$ (possibly $+\infty$ ).

Let $\mathcal{F}_{t}$ denote the $\sigma$-field generated by $(\xi(s): s \leq t)$. A direct application of Itô's formula shows that

$$
M_{t}:=\left(\hat{y}\left|g_{t}^{\prime}(\hat{z})\right| / y_{t}\right)^{a} \hat{G}\left(z_{t}\right)
$$

is a local martingale ${ }^{1}$.

As before, let $u_{t}=\log y_{t}$. Note that $w(u)=w_{t}:=x_{t} / y_{t}$ is a timehomogeneous diffusion process as a function of $u$. It is easy to see that $\tau(\hat{z})$ is a.s. the time at which $u$ hits $-\infty$. (For example, see the argument follow$\operatorname{ing}(3.2)$.)

If $t_{1}<\tau(\hat{z})$ is a stopping time, then by (6.3) we may write

$$
\log Z(\hat{z})=\int_{0}^{t_{1}}+\int_{t_{1}}^{\tau(\hat{z})} 4 y_{t}^{2}\left|z_{t}\right|^{-4} d t
$$

Note that if $s>0$ then the distribution of $Z(\hat{z})$ is the same as that of $Z(s \hat{z})$, by scale invariance. It is clear that for every $z^{\prime} \in \mathbb{H}$ there is positive probability that there will be some first time $t_{1}<\tau(\hat{z})$ such that $z_{t_{1}} /\left|z_{t_{1}}\right|=z^{\prime} /\left|z^{\prime}\right|$. Conditioned on $t_{1}<\tau(\hat{z})$ and on $\mathcal{F}_{t_{1}}$, the second integral in (6.4) has the same distribution as $\log Z\left(z^{\prime}\right)$, by the strong Markov property and scale invariance. Consequently, we find that $\mathbf{P}[Z(\hat{z})>s] \geq c \mathbf{P}\left[Z\left(z^{\prime}\right)>s\right]$ holds for every $s$, where $c=c\left(\hat{z}, z^{\prime}, \kappa\right)>0$. In fact, later we will need the following stronger

\footnotetext{
${ }^{1}$ The Markov property can be used to show that $\left(\hat{y}\left|g_{t}^{\prime}(\hat{z})\right| / y_{t}\right)^{a} G\left(z_{t}\right)$ is a local martingale. One then assumes that $G$ is smooth and applies Itô's formula, to obtain a PDE satisfied by $G$. Scale invariance can then be used to transform the PDE to the ODE (6.9) appearing below. Then one obtains $\hat{G}$ as a solution. That's how the function $\hat{G}$ was arrived at.
} 
statement (which is not needed for the present lemma, but we find it convenient to give the argument here). Let $\bar{w} \in(0, \infty)$. Then assuming $\left|w_{0}\right| \leq \bar{w}$, there is a $c=c(\bar{w}, \kappa)>0$ such that

$$
\forall s>0, \quad \mathbf{P}[Z(i)>s] \geq \mathbf{P}[Z(\hat{z})>s] \geq c \mathbf{P}[Z(i)>2 s] .
$$

The right-hand inequality is proved by taking $t_{1}$ to be the first time smaller than $\tau(z)$ such that $\operatorname{Re} z_{t_{1}}=0$ and the first integral in (6.4) is greater than $\log 2$, on the positive probability event that there is such a time. The lefthand inequality is clear, for if $\left|\operatorname{Re} g_{t}(i) / \operatorname{Im} g_{t}(i)\right|$ never hits $\left|w_{0}\right|$, then we have $Z(i)=\infty$, by $(6.3)$.

Note that $\hat{G}(i)=1$. Assume, for now, that $G(i)<\infty$. We claim that in this case $\hat{G}(x+i)>0$ for each $x \in \mathbb{R}$. Let $s_{0}>0$, let $T:=\inf \left\{t \geq 0:\left|w_{t}\right|=s_{0}\right\}$. Since $w(u)$ is time-homogeneous, clearly, $T<\tau(\hat{z})$. If $\hat{G}(x+i) \leq 0$ for some $x \in \mathbb{R}$, we may choose $s_{0}:=\inf \{x>0: \hat{G}(x+i)=0\}$. Then $M_{T}=0$ if $\left|w_{0}\right| \leq s_{0}$. Note that $1 \leq \hat{y}\left|g_{T}^{\prime}(\hat{z})\right| / y_{T}<Z$. Since $M_{t}$ is a local martingale on $t<\tau(\hat{z})$, there is an increasing sequence of stopping times $t_{n}<\tau(\hat{z})$ with $\lim _{n} t_{n}=\tau(\hat{z})$ a.s. such that $M_{t \wedge t_{n}}$ is a martingale. Set $\bar{t}_{n}:=T \wedge t_{n}$. The optional sampling theorem then gives for $\hat{z}=i$

$$
\begin{array}{r}
1=\hat{G}(\hat{z})=M_{0}=\mathbf{E}\left[M_{\bar{t}_{n}}\right]=\mathbf{E}\left[\left(\hat{y}\left|g_{\bar{t}_{n}}^{\prime}(\hat{z})\right| / y_{\bar{t}_{n}}\right)^{a} \hat{G}\left(z_{\bar{t}_{n}}\right)\right] \\
\leq \mathbf{E}\left[\max \left\{1, Z^{a}\right\} \hat{G}\left(z_{\bar{t}_{n}}\right)\right] .
\end{array}
$$

Since $\hat{G}\left(z_{t \wedge T}\right)$ is bounded, $\lim _{n \rightarrow \infty} \hat{G}\left(z_{\bar{t}_{n}}\right)=0$ a.s., and $\mathbf{E}\left[Z^{a}\right]<\infty$, we have $\lim _{n \rightarrow \infty} \mathbf{E}\left[\max \left\{1, Z^{a}\right\} \hat{G}\left(z_{\bar{t}_{n}}\right)\right]=0$, contradicting the above inequality. Consequently, $\hat{G}(x+i)>0$ for every $x \in \mathbb{R}$.

We maintain the assumption $G(i)<\infty$. By (6.5), this implies $G(z)<\infty$ for all $z \in \mathbb{H}$. Let $s_{1}, s_{2}, \ldots$ be a sequence in $(0, \infty)$ with $s_{n} \rightarrow \infty$ such that $L:=\lim _{n} \hat{G}\left(s_{n}+i\right)$ exists (allowing $L=\infty$ ). Now set $T_{m}:=\inf \left\{t \geq 0:\left|w_{t}\right|=\right.$ $\left.s_{m}\right\}$. The argument of the previous paragraph shows that $M_{0}=\mathbf{E}\left[M_{T_{m} \wedge t_{n}}\right] \rightarrow$ $\mathbf{E}\left[M_{T_{m}}\right]$, as $n \rightarrow \infty$. Therefore,

$$
\hat{G}(\hat{z})=M_{0}=\mathbf{E}\left[M_{T_{m}}\right] \rightarrow L G(\hat{z}), \quad m \rightarrow \infty,
$$

unless $L=\infty$, which happens if and only if $G(\hat{z})=0$. Moreover, $L$ does not depend on the particular sequence $s_{n}$, and we may write $\hat{G}(1)$ instead of $L$. Thus, when $G(i)<\infty$,

$$
G(\hat{z})=\hat{G}(\hat{z}) / \hat{G}(1)
$$

When $a=1-\kappa / 8, \hat{G}$ simplifies to $(y /|z|)^{(8-\kappa) / \kappa}$, with $\hat{G}(1)=\infty$ if $\kappa>8$ and $\hat{G}(1)=0$ if $\kappa<8$. It follows that $G(z)=\infty$ if $a=1-\kappa / 8$ and $\kappa<8$. Clearly, $G(z)=\infty$ also if $a>1-\kappa / 8$, and $\kappa<8$. Similarly, we have $G(z)=0$ if $\kappa>8$ and $a=1-\kappa / 8$. This implies that $Z=\infty$ a.s. when $\kappa>8$, which implies $G=0$ when $\kappa>8$ and $a<0$. There are various ways to argue that $Z=\infty$ a.s. when $\kappa=8$. In particular, it is easy to see that $\hat{G}_{\frac{1}{4}, 8}(1)=\infty$ 
(if $a_{n}$ denotes the $n$-th coefficient of ${ }_{2} F_{1}\left(\frac{1}{4}, \frac{1}{4}, \frac{1}{2}, z\right)$, then $(n+1) a_{n+1}=n a_{n} b_{n}$ with $b_{n}=(n+1 / 4)^{2} /(n(n+1 / 2))$ and it follows from $\prod_{n} b_{n}>0$ that $n a_{n}$ is bounded away from zero) so that the above argument applies. It remains to prove that $G(i)<\infty$ when $a<1-\kappa / 8$ and $\kappa<8$.

Assume now that $\kappa<8$ and $a<1-\kappa / 8$ and $a$ is sufficiently close to $1-\kappa / 8$ so that $\eta_{1}>0$. We prove below that in this range

$$
\inf _{z \in \mathbb{H}} \hat{G}(z)>0 \text {. }
$$

Since $\hat{G}(\hat{z})=M_{0}=\mathbf{E}\left[M_{t_{n}}\right]$ when $\hat{z}=i$, we immediately get $G(i)<\infty$ from (6.7). Since $G(i)<\infty$ for some $a$ implies $G(i)<\infty$ for any smaller $a$, it only remains to establish (6.7) in the above specified range.

To prove (6.7), first note that $\eta_{0} \leq \eta_{1}, 0<\eta_{1}<1 / 2$ and $\eta_{0}+\eta_{1}=$ $1-4 / \kappa<1 / 2$. By [EMOT53, 2.1.3.(14)], when $\eta_{2}>\eta_{1}+\eta_{0}$ and $\eta_{2}>\eta_{1}>0$, the right-hand side of (6.1) converges at $z=1$ and is equal to

$$
\frac{\Gamma\left(\eta_{2}\right) \Gamma\left(\eta_{2}-\eta_{1}-\eta_{0}\right)}{\Gamma\left(\eta_{2}-\eta_{1}\right) \Gamma\left(\eta_{2}-\eta_{0}\right)}
$$

This gives $\hat{G}(1)>0$. Now write

$$
g(x):=\hat{G}_{a, \kappa}(x+i) / \hat{G}_{1-\kappa / 8, \kappa}(x+i)=\left(1+x^{2}\right)^{(8-\kappa) /(2 \kappa)} \hat{G}_{a, \kappa}(x+i) .
$$

Since $\hat{G}$ satisfies

$$
\frac{4 a y^{2}}{|z|^{4}} \hat{G}+\frac{\kappa}{2} \partial_{x}^{2} \hat{G}+\frac{4 x}{|z|^{2}} \partial_{x} \hat{G}=0
$$

for $y=1$, it follows that

$$
(\kappa-8+8 a) g+2(\kappa-4) x\left(1+x^{2}\right) g^{\prime}+\kappa\left(1+x^{2}\right)^{2} g^{\prime \prime}=0 .
$$

It is immediate that $g(0)=1, g^{\prime}(0)=0$ and $g^{\prime \prime}(0)>0$, e.g., by differentiation or by considering (6.9). Consequently, $g>1$ for small $|x|>0$. Suppose that there is some $x \neq 0$ such that $g(x)=1$. Then there must be some $x_{0} \neq 0$ such that $g\left(x_{0}\right)>1$ and $g$ has a local maximum at $x_{0}$. Then $g^{\prime}\left(x_{0}\right)=0 \geq g^{\prime \prime}\left(x_{0}\right)$. But this contradicts (6.10), giving $g>1$ for $x \neq 0$. Together with $\hat{G}(1)>0$, this implies (6.7), and completes the proof.

We say that $z \in \overline{\mathbb{H}}$ is swallowed by the SLE if $z \notin \gamma[0, \infty)$ but $z \in K_{t}$ for some $t \in(0, \infty)$. The smallest such $t$ will be called the swallowing time of $z$.

TheOREM 6.4. If $\kappa \in(4,8)$ and $z \in \overline{\mathbb{H}} \backslash\{0\}$, then a.s. $z$ is swallowed. If $\kappa \notin(4,8)$, then a.s. no $z \in \overline{\mathbb{H}} \backslash\{0\}$ is swallowed.

Lemma 6.5. Let $z \in \overline{\mathbb{H}} \backslash\{0\}$. If $\kappa>4$, then $\mathbf{P}[\tau(z)<\infty]=1$.

In fact, in this range $\kappa>4$, the stronger statement that a.s. for all $z \in \overline{\mathbb{H}}$ we have $\tau(z)<\infty$ holds as well. This follows from Theorem 7.1 (and its extension to $\kappa=8$ based on [LSW]). 
Proof. Let $b:=1-4 / \kappa$, and $\theta:=\exp (i \pi(1-b) / 2)$. Set

$$
h(z):=\operatorname{Im}\left(\theta z^{b}\right), \quad z \in \overline{\mathbb{H}} .
$$

A direct calculation shows that the drift term in Itô's formula for $\left(g_{t}(z)-\xi(t)\right)^{b}$ is zero. Consequently, $h\left(g_{t}(z)-\xi(t)\right)$ is a local martingale. Note that $\kappa>4$ implies that $h(z) \rightarrow \infty$ as $z \rightarrow \infty$ in $\overline{\mathbb{H}}$, that $h(z)>0$ in $\overline{\mathbb{H}} \backslash\{0\}$ and $h(0)=0$.

Fix some $z_{0} \in \mathbb{H}$ and some $\varepsilon>0$. For $t \geq 0$ set $z_{t}:=g_{t}\left(z_{0}\right)-\xi(t)$. We shall prove that there is some $T=T\left(z_{0}, \varepsilon\right)$ such that $\mathbf{P}\left[z_{0} \in K_{T}\right]>1-\varepsilon$. Indeed, let $R>1$ be sufficiently large so that $\varepsilon \inf \{h(z):|z|=R\}>2 h\left(z_{0}\right)$. Let

$$
\tau_{R}:=\inf \left(\left\{t \in\left[0, \tau\left(z_{0}\right)\right):\left|z_{t}\right| \geq R\right\} \cup\left\{\tau\left(z_{0}\right)\right\}\right) .
$$

We claim that if $T=T\left(z_{0}, \varepsilon\right)$ is sufficiently large, then $\mathbf{P}\left[\tau_{R}>T\right]<\varepsilon / 2$. Let $t \geq 0$ and let $\lceil t\rceil:=\inf \{k \in \mathbb{Z}: k \geq t\}$. Say that $t$ is a sprint time if $|\xi(t)-\xi(\lceil t\rceil)|=R$ and there is no $s \in[t,\lceil t\rceil]$ such that $|\xi(s)-\xi(\lceil t\rceil)|=R$. Note that for every integer $k \geq 0$ there is positive probability that there is a sprint time in $[k, k+1]$ and this event is independent from $(\xi(t): t \in[0, k])$. Therefore, there are infinitely many sprint times a.s. Let $t_{0}<t_{1}<t_{2}<\cdots$ be the sequence of sprint times. Let $\mathcal{A}_{j}$ be the event that $\xi\left(\left\lceil t_{j}\right\rceil\right)>\xi\left(t_{j}\right)$. Observe that for all $j \in \mathbb{N}$,

$$
\mathbf{P}\left[\mathcal{A}_{j} \mid t_{j}, \mathcal{F}_{t_{j}}\right]=1 / 2,
$$

where $\mathcal{F}_{t}$ denotes the $\sigma$-field generated by $(\xi(s): s \in[0, t])$, because given $t_{j}$ and $\left(\xi(t): t \in\left[0, t_{j}\right]\right)$, the distribution of $\left(\xi(t): t>t_{j}\right)$ is the same as that of $\left(2 \xi\left(t_{j}\right)-\xi(t): t>t_{j}\right)$, by reflection symmetry. Let $x_{t}:=\operatorname{Re}\left(z_{t}\right)$, and note that

$$
d x_{t}=2 x_{t}\left|z_{t}\right|^{-2} d t-d \xi(t) .
$$

Consequently, for $s \geq t_{j}$,

$$
x_{s}=x_{t_{j}}+2 \int_{t_{j}}^{s} x_{t}\left|z_{t}\right|^{-2} d t-\xi(s)+\xi\left(t_{j}\right) .
$$

On $\mathcal{A}_{j}$ we have $\xi(s)-\xi\left(t_{j}\right)>0$ for all $s \in\left[t_{j},\left[t_{j}\right]\right]$. Hence, it follows from (6.12) that on $\mathcal{A}_{j}$ and assuming $x_{t_{j}} \leq 0$, we have $x_{s} \leq 0$ for all $s \in\left[t_{j},\left\lceil t_{j}\right\rceil\right]$ and moreover $x_{\left\lceil t_{j}\right\rceil}<-R$. Similarly, if $x_{t_{j}} \geq 0$ and $\neg \mathcal{A}_{j}$ holds, then $x_{\left\lceil t_{j}\right\rceil}>R$. Hence, by (6.11) we have $\mathbf{P}\left[\tau_{R}>\left\lceil t_{j}\right\rceil\right] \leq 2^{-j}$, which implies that we may choose $T$ such that $\mathbf{P}\left[\tau_{R}>T\right]<\varepsilon / 2$. Set $\tau^{*}:=\tau_{R} \wedge T$, where $T$ is as above.

Since $h\left(z_{t}\right)$ is a local martingale, and $h\left(z_{t \wedge \tau^{*}}\right)$ is bounded, $h\left(z_{t \wedge \tau^{*}}\right)$ is a martingale. Therefore,

$$
h\left(z_{0}\right)=\mathbf{E}\left[h\left(z_{\tau^{*}}\right)\right] \geq \inf \{h(z):|z|=R\} \mathbf{P}\left[\left|z_{\tau^{*}}\right|=R\right] .
$$

By our choice of $R$, this gives $\mathbf{P}\left[\left|z_{\tau^{*}}\right|=R\right]<\varepsilon / 2$. Since $\mathbf{P}\left[\tau_{R}>T\right]<\varepsilon / 2$, it follows that $\mathbf{P}\left[\tau\left(z_{0}\right) \leq T\right] \geq 1-\varepsilon$, and the lemma is thereby established. 
We now prove a variant of Cardy's formula. (See [Car] for a survey of Cardy's formula.) A proof of (a generalized) Cardy's formula for $\mathrm{SLE}_{\kappa}$ appears in [LSW01a]. However the following variant appears to be different, except in the special case $\kappa=6$, in which they are equivalent due to the locality property of $\mathrm{SLE}_{6}$. (See [LSW01a] for an explanation of the locality property.)

LEMma 6.6. Fix $\kappa \in(4,8)$ and let

$$
X:=\inf ([1, \infty) \cap \gamma[0, \infty)) .
$$

Then for all $s \geq 1$,

$$
\mathbf{P}[X \geq s]=\frac{4^{(\kappa-4) / \kappa} \sqrt{\pi}_{2} F_{1}(1-4 / \kappa, 2-8 / \kappa, 2-4 / \kappa, 1 / s) s^{(4-\kappa) / \kappa}}{\Gamma(2-4 / \kappa) \Gamma(4 / \kappa-1 / 2)}
$$

The proof will be brief, since it is similar to the detailed proof of the more elaborate result in [LSW01a].

Proof. Let $s>1$, and set

$$
Y_{t}:=\frac{g_{t}(1)-\xi(t)}{g_{t}(s)-\xi(t)}
$$

for $t<\tau(1)$. It is not hard to see that $\lim _{t / \tau(1)} Y_{t}=1$ if $X>s$ and $\lim _{t \nearrow \tau(1)} Y_{t}=0$ if $X<s$ : Just notice that for $X>s$ and $t \nearrow \tau(1)$, the extremal distance within $H_{t}$ from $[1, s]$ to $[\operatorname{Re} \gamma(t), \gamma(t)]$ tends to $\infty$, whereas for $X<s$ we have $\tau(1)<\tau(s)$. Direct inspection shows that the right-hand side of (6.13) is zero when $s=\infty$ and is 1 when $s=1$. In particular, it is bounded for $s \in[1, \infty)$. Moreover, Itô's formula shows that when we plug $1 / Y_{t}$ in place of $s$ in the right-hand side of (6.13), we get a local martingale. Since $Y_{0}=1 / s$ and $Y_{t} \in(0,1)$ for $t<\tau(1)$, it therefore suffices to prove that $\lim _{t / \tau(1)} Y_{t} \in\{0,1\}$ a.s. The only way this could fail is if $X=s$ occurs with positive probability. However, scale invariance shows that $\mathbf{P}\left[X=s^{\prime}\right] \geq \mathbf{P}[X=s]$ when $s^{\prime} \in(1, s)$. Hence $\mathbf{P}[X=s]=0$ for all $s \neq 1$.

Proof of Theorem 6.4. The case $\kappa \in[0,4]$ is covered by Theorem 6.1 and Theorem 5.1.

Suppose that $\kappa \in(4,8)$ and $z \in \overline{\mathbb{H}}$. Lemma 6.5 shows that $z \in \bigcup_{t>0} K_{t}$ a.s. If $z \in \mathbb{H}$, then Lemma 6.3 and $(6.2)$ show a.s. $z \notin \gamma[0, \infty)$. Lemma 6.6 shows that $1 \notin \gamma[0, \infty)$ a.s., and the same follows for every $z \in \mathbb{R} \backslash\{0\}$ by scale and reflection invariance. This covers the range $\kappa \in(4,8)$.

Now suppose that $z_{0}$ is swallowed, and let $t_{0}$ be the swallowing time. If $\delta>0$ is smaller than the distance from $z_{0}$ to $\gamma\left[0, t_{0}\right]$, then all the points within distance $\delta$ from $z_{0}$ are swallowed with $z_{0}$. Therefore, if the probability that some $z_{0} \in \overline{\mathbb{H}} \backslash\{0\}$ is swallowed is positive, then by (6.2) there is some $z_{0}$ such that $\mathbf{P}\left[Z\left(z_{0}\right)<\infty\right]>0$. By Lemma 6.3 , this implies that for $\kappa \geq 8$ a.s. there is no $z \in \overline{\mathbb{H}} \backslash\{0\}$ which is swallowed. 
Exercise 6.7. Prove that $\mathbf{P}[\tau(z)<\infty]=0$ when $\kappa \in(0,4]$ and $z \in \mathbb{H}$.

\section{Transience}

THEOREM 7.1 (Transience). For all $\kappa \neq 8$ the $\operatorname{SLE}_{\kappa}$ trace $\gamma(t)$ is transient a.s. That is,

$$
\mathbf{P}\left[\liminf _{t \rightarrow \infty}|\gamma(t)|=\infty\right]=1
$$

Lemma 7.2. Suppose that $\kappa \leq 4$, and let $x \in \mathbb{R} \backslash\{0\}$. Then a.s. $x \notin$
$\overline{\gamma[0, \infty)}$.

Proof. By symmetry, we may assume that $x>0$. Moreover, scaling shows that it is enough to prove the lemma in the case $x=1$. So assume that $x=1$. Let $s \in(0,1 / 4)$ and suppose that there is some first time $t_{0}>0$ such that $\left|\gamma\left(t_{0}\right)-1\right|=s$. This implies that $\left|g_{t_{0}}(1 / 2)-\xi\left(t_{0}\right)\right|=O(s)$. Indeed, $\left|g_{t_{0}}(1 / 2)-\xi\left(t_{0}\right)\right|$ is the limit as $y \rightarrow \infty$ of $y$ times the harmonic measure in $\mathbb{H} \backslash \gamma\left[0, t_{0}\right]$ from $i y$ of the union of $[0,1 / 2]$ and the "right-hand side" of $\gamma\left[0, t_{0}\right]$. By the maximum principle, this harmonic measure is bounded above by the harmonic measure in $\mathbb{H}$ of the interval $\left[1, \gamma\left(t_{0}\right)\right]$, which is $O(s / y)$.

In the proof of Lemma 6.2, we have determined the probability that $Y_{x}(t)=g_{t}(x)-\xi(t)$ hits $b$ before $a$, where $x \in[a, b]$ and $0<a<b$. For $\kappa<4$, that probability goes to 1 as $a \searrow 0$, uniformly in $b$. Consequently, a.s. there is a random $\varepsilon>0$ such that for all $b>x, Y_{x}(t)$ hits $b$ before $\varepsilon$. Since $\sup _{t \in[0, T]} Y_{x}(t)<\infty$ a.s. for all finite $T$, it follows that $\inf _{t \geq 0} Y_{x}(t)>0$ a.s. Using this with $x=1 / 2$, we conclude from the previous paragraph that a.s. $1 \notin \overline{\gamma[0, \infty)}$, at least when $\kappa<4$.

Assume now that $\kappa=4$. Although the above proof does not apply (since $Y_{x}(t)$ is recurrent), there are various different ways to handle this case. Let $0<y<x<\infty$. Let $\bar{\gamma}(t)$ denote the complex conjugate of $\gamma(t)$, and set $D_{t}:=\mathbb{C} \backslash(\gamma[0, t] \cup \bar{\gamma}[0, t] \cup(-\infty, y])$. Using Schwarz reflection, $g_{t}$ may be conformally extended to $D_{t}$. Note that $g_{t}\left(D_{t}\right)=\mathbb{C} \backslash\left(-\infty, g_{t}(y)\right]$. By the Koebe $1 / 4$ Theorem applied to $g_{t}^{-1}$, the distance from $x$ to $\partial D_{t}$ is at least $\frac{1}{4}\left(g_{t}(x)-g_{t}(y)\right) / g_{t}^{\prime}(x)$. Consequently, it suffices to show that a.s.

$$
\sup _{t \geq 0} g_{t}^{\prime}(x) /\left(g_{t}(x)-g_{t}(y)\right)<\infty \text {. }
$$

Set $Q(t)=Q_{x, y}(t):=\log g_{t}^{\prime}(x)-\log \left(g_{t}(x)-g_{t}(y)\right)$. Then the above reduces to verifying that $\sup _{t} Q(t)<\infty$. The time derivative of $Q$ is

$$
\partial_{t} Q(t)=-2 Y_{x}(t)^{-2}+2 Y_{x}(t)^{-1} Y_{y}(t)^{-1} .
$$

In particular, $Q(t)$ is increasing in $t$. Define

$$
G(s):=\log s \log (1+s)-\frac{1}{2} \log ^{2}(1+s)+\operatorname{Li}_{2}(-s),
$$


where $\operatorname{Li}_{2}(z)=\int_{z}^{0} s^{-1} \log (1-s) d s$. Note that $G$ satisfies $s(1+s)^{2} G^{\prime \prime}(s)+$ $s(1+s) G^{\prime}(s)=1$. A straightforward application of Itô's formula shows that $Q(t)-G_{t}$ is a local martingale, where

$$
G_{t}:=G\left(\frac{g_{t}(x)-g_{t}(y)}{g_{t}(y)-\xi(t)}\right)
$$

Consequently, there is an increasing sequence of stopping times $t_{n}$ tending to $\infty$ a.s. such that $\mathbf{E}\left[Q\left(t_{n}\right)\right]=\mathbf{E}\left[G_{t_{n}}\right]+Q(0)-G_{0}$. It is immediate to verify that $\sup \{G(x): x>0\}<\infty$. Hence, $\lim _{\sup _{n \rightarrow \infty}} \mathbf{E}\left[Q\left(t_{n}\right)\right]<\infty$. Because $Q$ is increasing in $t$, it follows that $\mathbf{E}\left[\sup _{t} Q(t)\right]<\infty$ and hence $\sup _{t} Q(t)<\infty$ a.s., which completes the proof.

LEMma 7.3. Suppose that $\kappa>4, \kappa \neq 8$, and let $t>0$. Then a.s. there is some $\varepsilon>0$ such that $K_{t} \supset\{z \in \mathbb{H}:|z|<\varepsilon\}$.

Proof. Consider first the case $\kappa \in(4,8)$. We know that $\gamma(t)$ is a continuous path, by Theorem 5.1. Note that by scale and reflection invariance, Lemma 6.6 shows that for $a<b<0$ there is a positive probability that the interval $[a, b]$ is swallowed all at once; i.e., $\mathbf{P}[[a, b] \cap \gamma[0, \infty)=\emptyset]>0$, but a.s. there is some $t$ such that $[a, b] \subset K_{t}$. When this happens, we have $[a, b] \cap \overline{H_{t}}=\emptyset$.

Let $S$ be the set of limit points of $g_{\tau(1)}(z)$ as $z \rightarrow 0$ in $H_{\tau(1)}$. (If $0 \notin \overline{H_{\tau(1)}}$, set $S=\emptyset$.) Then $S \subset(-\infty, \xi(\tau(1)))$. Note that $\left(g_{\tau(1)}(\gamma(t+\tau(1)))-\xi(\tau(1))\right.$ : $t \geq 0)$ has the same distribution as $(\gamma(t): t \geq 0)$. Consequently, by the above paragraph, there is positive probability that $g_{\tau(1)}(\gamma[\tau(1), \infty))$ is disjoint from $S$, but there is some $t>\tau(1)$ such that $g_{\tau(1)}\left(K_{t} \backslash K_{\tau(1)}\right) \supset S$. This implies that with positive probability there is some $t>0$ such that

$$
\delta:=\mathbf{P}\left[0 \notin \overline{H_{t}}\right]>0 .
$$

By scale invariance, $\delta$ does not depend on $t$. Since these events are monotone in $t$, it follows that

$$
\mathbf{P}\left[\forall t>0,0 \notin \overline{H_{t}}\right]=\delta
$$

By Blumenthal's 0-1 law it therefore follows that $\delta=1$. This completes the proof in the case $\kappa \in(4,8)$.

Now suppose that $\kappa>8$. The above argument implies that it is sufficient to show that there is some $t>0$ such that with positive probability $0 \notin \overline{H_{t}}$. Striving for a contradiction, we assume that for all $t>0$ a.s. $0 \in \overline{H_{t}}$.

Let $z_{0} \in \mathbb{H}$ be arbitrary. We know from Theorem 6.4 and Lemma 6.5 that a.s. $z_{0} \in \gamma[0, \infty)$. Set $t_{0}:=\tau\left(z_{0}\right)$. Then $z_{0}=\gamma\left(t_{0}\right)$. Let $t>0$. Conditioned on $\mathcal{F}_{t_{0}}$, the set $g_{t_{0}}\left(K_{t+t_{0}} \backslash K_{t_{0}}\right)$ has the same distribution as $K_{t}+\xi\left(t_{0}\right)$. It therefore follows by our above assumption that a.s. $\xi\left(t_{0}\right) \in \overline{g_{t_{0}}\left(H_{t+t_{0}}\right)}$. Mapping by $g_{t_{0}}^{-1}$, this implies that a.s. $z_{0} \in \overline{H_{t+t_{0}}}$; that is, $z_{0} \in \partial K_{t+t_{0}}$. We conclude that for 
every $z \in \mathbb{H}$ and every $t>0$ a.s. $z \in \partial K_{t+\tau(z)}$. Therefore, a.s.

$$
\operatorname{area}\left(\partial K_{t}\right)=\int_{z \in \mathbb{H}} 1_{z \in \partial K_{t}} d x d y \geq \int_{z \in \mathbb{H}} 1_{t>\tau(z)} d x d y,
$$

and Fubini implies that $\partial K_{t}$ has positive measure with positive probability for large $t$. However, this contradicts Corollary 5.3, completing the proof of the lemma.

Proof of Theorem 7.1. First suppose that $\kappa>4$. Then we know from Lemma 7.3 that there is a random but positive $r>0$ such that $K_{1} \supset$ $\{z \in \mathbb{H}:|z|<r\}$. Let $\varepsilon>0$, and let $r_{0}=r_{0}(\kappa, \varepsilon)>0$ be a constant such that $\mathbf{P}\left[r<r_{0}\right]<\varepsilon$. Then the scaling property of SLE shows that for all $t>0$

$$
\mathbf{P}\left[K_{t} \supset\left\{z \in \mathbb{H}:|z|<r_{0} \sqrt{t}\right\}\right] \geq 1-\varepsilon .
$$

This implies that

$$
\mathbf{P}\left[\exists t^{\prime}>t:\left|\gamma\left(t^{\prime}\right)\right|<r_{0} \sqrt{t}\right]<\varepsilon .
$$

Since $\varepsilon>0$ was arbitrary, this clearly implies transience.

Consider now the case $\kappa \in[0,4]$. In this range, $\gamma$ is a simple path. Then a.s. there are two limit points $x_{0}, x_{1}$ for $g_{1}(z)$ as $z \rightarrow 0$ in $H_{1}$. Note that $g_{1}(\gamma[1, \infty))$ has the same distribution as $\gamma[0, \infty)$ translated by $\xi(1)$. Consequently, Lemma 7.2 shows that a.s. $x_{0}$ and $x_{1}$ are not in the closure of $g_{1}(\gamma[1, \infty))$. This means that $0 \notin \overline{\gamma[1, \infty)}$ a.s. The proof is then completed as in the case $\kappa>4$ above.

Corollary 7.4. Suppose that $\kappa>8$, then $\gamma[0, \infty)=\overline{\mathbb{H}}$ a.s.

Proof. From Lemma 6.3 and $(6.2)$ we know that $\gamma[0, \infty)$ is a.s. dense in $\overline{\mathbb{H}}$. Since $\gamma$ is a.s. transient, $\gamma[0, \infty)$ is closed in $\mathbb{C}$, and hence is equal to $\overline{\mathbb{H}}$.

Update. Corollary 7.4 and Theorem 7.1 are true also for $\kappa=8$. The proofs are based on the extension [LSW] to $\kappa=8$ of Theorem 5.1, and are otherwise the same.

\section{Dimensions}

In this section we study the size of the SLE trace and the hull boundary. When $\kappa \leq 4$, these two sets are the same, and it is interesting to compare the methods and results. For sets $A \subset \mathbb{C}$, denote by $N(\varepsilon)=N(\varepsilon, A)$ the minimal number of disks of radius $\varepsilon$ needed to $\operatorname{cover} A$. 
8.1. The size of the trace.

TheOREM 8.1. For every nonempty bounded open set $A$ with $\bar{A} \subset \mathbb{H}$,

$$
\limsup _{\varepsilon \rightarrow 0} \frac{\log \mathbf{E}[N(\varepsilon, \gamma[0, \infty) \cap A)]}{|\log \varepsilon|}= \begin{cases}1+\kappa / 8 & \kappa<8, \\ 2 & \kappa \geq 8 .\end{cases}
$$

Proof. We may assume $\kappa<8$ because $\gamma[0, \infty)=\overline{\mathbb{H}}$ if $\kappa>8$, by Corollary 7.4 , and when $\kappa=8$ we know that for every $z \in \mathbb{H}$ we have $z \in \gamma[0, \infty)$ a.s., by Theorem 6.4 and Lemma 6.5. Fix $\varepsilon>0$ smaller than the distance from $A$ to $\mathbb{R}$, and denote those points $z \in \varepsilon \mathbb{Z}^{2}$ with $\operatorname{dist}(z, A)<\varepsilon$ by $z_{j}, 1 \leq j \leq n$. Then $n$ is comparable to $1 / \varepsilon^{2}$. Set $N:=N(\varepsilon, \gamma[0, \infty) \cap A)$. Obviously $N \leq \#\left\{z_{j}: \operatorname{dist}\left(z_{j}, \gamma[0, \infty)\right)<\varepsilon\right\} \leq 100 N$. From (6.2) and (6.5) we get that $\mathbf{E}[N]$ is comparable to

$$
\sum_{j=1}^{n} \mathbf{P}\left[Z\left(z_{j}\right)>\varepsilon^{-1}\right]
$$

Write $F(t)=\mathbf{P}[Z(i)>t]$. Using (6.5), the proof of the theorem reduces to showing that

$$
\limsup _{t \rightarrow \infty} \frac{\log F(t)}{\log t}=\kappa / 8-1
$$

By (6.8),

$$
\lim _{a / 1-\kappa / 8} \frac{\hat{G}_{a, \kappa}(1)}{1-\kappa / 8-a}=\frac{\sqrt{\pi} \Gamma(4 / \kappa-1 / 2)}{2 \Gamma(4 / \kappa)}
$$

and since $\hat{G}_{a, \kappa}(i)=1$ from Lemma 6.3 we obtain

$$
C^{-1} \frac{1}{1-\kappa / 8-a} \leq \mathbf{E}\left[Z(i)^{a}\right] \leq C \frac{1}{1-\kappa / 8-a}
$$

for all $a<1-\kappa / 8$ sufficiently close to $1-\kappa / 8$. Hence, for small $\Delta=\Delta(\kappa)>0$,

$$
C \Delta \geq \int_{0}^{\Delta} \mathbf{E}\left[Z(i)^{1-\kappa / 8-\delta}\right] \delta d \delta=\int_{0}^{\Delta} \int_{0}^{\infty}(1-\kappa / 8-\delta) t^{-\kappa / 8-\delta} F(t) d t \delta d \delta .
$$

From $\int_{0}^{\Delta} t^{-\kappa / 8-\delta} \delta d \delta \geq C t^{-\kappa / 8} /(\log t)^{2}$ for $t \geq e$ (where $\Delta$ is treated as a constant), we obtain

$$
\int_{e}^{\infty} \frac{t^{-\kappa / 8} F(t)}{(\log t)^{2}} d t=O(1) .
$$

Using $F(t) / F(2 t)=O(1)$ from (6.5) this easily implies for large $t$

$$
F(t) \leq C t^{\kappa / 8-1}(\log t)^{2} .
$$

There is a sequence $t_{n} \rightarrow \infty$ with

$$
F\left(t_{n}\right) \geq \frac{t_{n}^{\kappa / 8-1}}{\left(\log t_{n}\right)^{2}},
$$


for otherwise we would have $\mathbf{E}\left[Z(i)^{1-\kappa / 8}\right]=O(1) \int_{e}^{\infty} \frac{d t}{t(\log t)^{2}}<\infty$, contradicting Lemma 6.3.

An immediate consequence is

Corollary 8.2. For $\kappa<8$, the Hausdorff dimension of $\gamma[0, \infty)$ is a.s. bounded above by $1+\kappa / 8$.

8.2. The size of the hull boundary. When studying the size of the hull boundary $\partial K_{t}$, it suffices to restrict to $t=1$, by scaling. We will first estimate the size of $\partial H_{1} \cap\{y>h\}$ for small $h>0$ in terms of the convergence exponent of the Whitney decomposition of $H_{1}$. A Whitney decomposition of $H_{1}$ is a covering by essentially disjoint closed squares $Q \subset H_{1}$ with sides parallel to the coordinate axes such that the side length $d(Q)$ is comparable to the distance of $Q$ from the boundary of $H_{1}$. One can always arrange the side lengths to be integer powers of 2 and that

$$
d(Q) \leq \operatorname{dist}\left(Q, \partial H_{1}\right) \leq 8 d(Q) .
$$

For example, one can take all squares of the form $Q=2^{n}([k, k+1] \times[j, j+1])$ such that $Q \subset H_{1}, d(Q) \leq \operatorname{dist}\left(Q, \partial H_{1}\right)$, and $Q$ is not strictly contained in any other $Q^{\prime}$ satisfying these conditions. One important feature of the Whitney decomposition is that the hyperbolic diameters of the tiles in the decomposition are bounded away from 0 and $\infty$. (The hyperbolic metric on $H_{1}$ is the metric for which the conformal map $g_{1}: H_{1} \rightarrow \mathbb{H}$ is an isometry, where $\mathbb{H}$ is taken with the hyperbolic metric with length element $y^{-1}|d z|$.) This follows from the well-known consequence of the Koebe $1 / 4$ theorem saying that the hyperbolic length element in $H_{1}$ is comparable to $\operatorname{dist}\left(z, \partial H_{1}\right)^{-1}|d z|$. This description of the hyperbolic metric will be useful below.

Fix a Whitney decomposition of $H_{1}$, and denote by $W$ the collection of those squares $Q$ for which $\operatorname{dist}\left(Q, K_{1}\right) \leq 1$ and $\sup \{\operatorname{Im} z: z \in Q\} \geq h$. For $a>0$ let

$$
S(a)=S_{h}(a):=\sum_{Q \in W} d(Q)^{a} .
$$

Theorem 8.3. Set

$$
\delta(\kappa):= \begin{cases}1+\kappa / 8 & \kappa \leq 4 \\ 1+2 / \kappa & \kappa>4\end{cases}
$$

For $\kappa>0, \kappa \neq 4$, and all sufficiently small $h \in(0,1)$ we have

$$
\mathbf{E}[S(a)] \begin{cases}<\infty & a>\delta(\kappa), \\ =\infty & a \leq \delta(\kappa) .\end{cases}
$$

For $j, n \in \mathbb{Z}$, let $x_{j, n}:=j 2^{-n}, y_{n}:=2^{-n}$ and $z_{j, n}=x_{j, n}+i y_{n}$. Let $I$ be the interval $g_{1}\left(\partial K_{1}\right)-\xi(1)$; that is, $\hat{f}^{-1}\left(\partial K_{1}\right)$. 
LEMMA 8.4. There are constants $C=C(h), c=c(h)>0$ such that the following holds. Every $Q \in W$ is within distance $C$ in the hyperbolic metric of $H_{1}$ from some $\hat{f}_{1}\left(z_{j, n}\right)$ with $x_{j, n} \in I$ and $n \geq 0$, and every $\hat{f}_{1}\left(z_{j, n}\right)$ such that $x_{j, n} \in I, n \geq 0$ and $\operatorname{Im} \hat{f}_{1}\left(z_{j, n}\right) \geq h$ is within hyperbolic distance $C$ from some $Q \in W$. Moreover, in each of these cases,

$$
c^{-1} d(Q) \leq y_{n}\left|\hat{f}_{1}^{\prime}\left(z_{j, n}\right)\right| \leq c d(Q)
$$

Proof. Observe that the diameter of $K_{1}$ is bounded from below, because the uniformizing map $g: \mathbb{H} \backslash K \rightarrow \mathbb{H}$ with the hydrodynamic normalization (2.2) tends to the identity as $K$ tends to a point, and the uniformizing map $g_{1}$ for $K_{1}$ satisfies $g_{1}(z)=z+2 / z+\cdots$ near $\infty$, by $(2.3)$.

Let $\hat{z} \in Q \in W$. It follows from the definition of $W$ and the fact that $\operatorname{diam}\left(K_{1}\right)$ is bounded from zero that the harmonic measure of $K_{1}$ from $\hat{z}$ in $H_{1}$ is bounded from zero. Therefore, the harmonic measure of $I$ from $\hat{f}_{1}^{-1}(\hat{z})$ in $\mathbb{H}$ is bounded from zero. This implies that there is some $z_{j, n}$ within bounded distance from $\hat{f}_{1}^{-1}(\hat{z})$ in the hyperbolic metric of $\mathbb{H}$ such that $x_{j, n} \in I$. Then $\hat{f}_{1}\left(z_{j, n}\right)$ is within bounded hyperbolic distance from $Q$. Because $\sup \{\operatorname{Im} z$ : $\left.z \in K_{1}\right\}=O(1)$, we also have $\operatorname{Im} \hat{z}=O(1)$, and therefore $\operatorname{Im} \hat{f}_{1}\left(z_{j, n}\right)=$ $O(1)$. Since $\operatorname{Im} \hat{f}_{1}\left(z_{j, n}\right) \geq \operatorname{Im} z_{j, n}$, it follows that $\max \{0,-n\}=O(1)$. Then $z^{\prime}:=x_{j, n}+i 2^{n \wedge 0}$ is equal to $z_{j^{\prime}, n^{\prime}}$ for some $j^{\prime} \in \mathbb{Z}, n^{\prime} \in \mathbb{N}$, and satisfies the requirements in the first statement of the lemma.

From the fact that $\operatorname{diam}\left(K_{1}\right)$ is bounded from zero it also follows that $|I|$, the length of $I$, is bounded from zero. Suppose that we have $x_{j, n} \in I$, $j \in \mathbb{Z}, n \in \mathbb{N}$. Then the harmonic measure of $I$ from $z_{j, n}$ is bounded from zero. Consequently, the harmonic measure of $K_{1}$ from $\hat{f}_{1}\left(z_{j, n}\right)$ in $H_{1}$ is bounded from zero. Now suppose also that $\operatorname{Im} \hat{f}_{1}\left(z_{j, n}\right) \geq h$. Since $\sup \left\{\operatorname{Im} z: z \in K_{1}\right\}=O(1)$ and $\operatorname{Im} \hat{f}_{1}\left(z_{j, n}\right)=O(1)$, it follows that the Euclidean distance from $\hat{f}_{1}\left(z_{j, n}\right)$ to $K_{1}$ is bounded from above. If $\operatorname{dist}\left(\hat{f}_{1}\left(z_{j, n}\right), K_{1}\right) \leq 1$, then the Whitney tile $Q$ containing $\hat{f}_{1}\left(z_{j, n}\right)$ is in $W$. Otherwise, let $p$ be a point closest to $\hat{f}_{1}\left(z_{j, n}\right)$ among the points $z$ satisfying $\operatorname{Im} z \geq h$ and $\operatorname{dist}\left(z, K_{1}\right) \leq 1$. Since $h<1$ and $\operatorname{dist}\left(\hat{f}_{1}\left(z_{j, n}\right), K_{1}\right)=O(1)$, it follows that $\left|p-\hat{f}_{1}\left(z_{j, n}\right)\right|=\bar{O}(1)$. Since the points in $\left[p, \hat{f}_{1}\left(z_{j, n}\right)\right]$ have distance at least $h$ from $\partial H_{1}$, it follows that the hyperbolic distance from $p$ to $\hat{f}_{1}\left(z_{j, n}\right)$ is bounded above. For the second statement of the lemma we may then take $Q$ to be the Whitney tile containing $p$.

The last statement holds because $\hat{f}_{1}$ is an isometry from the hyperbolic metric of $\mathbb{H}$ to the hyperbolic metric of $H_{1}$.

Proof of Theorem 8.3. Set

$$
\tilde{S}_{h}(a):=\sum_{j=-\infty}^{\infty} \sum_{n=0}^{\infty} 1_{x_{j, n} \in I} 1_{\operatorname{Im} \hat{f}_{1}\left(z_{j, n}\right)>h}\left|\hat{f}_{1}^{\prime}\left(z_{j, n}\right) y_{n}\right|^{a}
$$


We now show that this quantity is comparable to $S(a)$. Let $C$ be the constant in the lemma. Since each square $Q \in W$ has a bounded diameter in the hyperbolic metric of $H_{1}$, and the hyperbolic distance in $\mathbb{H}$ between any two distinct $z_{j, n}$ is bounded from below, it follows that there are at most a bounded number of $\hat{f}_{1}\left(z_{j, n}\right)$ within distance $C$, in the hyperbolic metric of $H_{1}$, from any single $Q \in W$. Similarly, there are at most a bounded number of $Q \in W$ within hyperbolic distance $C$ from any point in $H_{1}$. Moreover, if $z$ is within hyperbolic distance $C$ from some $Q \in W$, then $\operatorname{Im} z$ is bounded away from zero, because the hyperbolic distance in $H_{1}$ between any two points is an upper bound on the distance between them in the hyperbolic metric of $\mathbb{H}$ (by the Schwarz lemma, for instance). Consequently, Lemma 8.4 implies

$$
c_{1}^{-1} \tilde{S}_{h}(a) \leq S_{h}(a) \leq c_{1} \tilde{S}_{h^{\prime}}(a),
$$

for some constant $c_{1}, h^{\prime}$, which depends only on $h$ and $a$. Now,

$$
\mathbf{E}\left[\tilde{S}_{h}(a)\right]=\sum_{n=0}^{\infty} \sum_{m=0}^{\infty} \sum_{j=-\infty}^{\infty} \mathbf{E}\left[\left|\hat{f}_{1}^{\prime}\left(z_{j, n}\right) y_{n}\right|^{a} 1_{\mathcal{A}(j, n, m)}\right]
$$

where $\mathcal{A}(j, n, m)$ denotes the event $\left\{x_{j, n} \in I, \operatorname{Im} \hat{f}_{1}\left(z_{j, n}\right) \geq h, m \leq|I|<m+1\right\}$. We split this sum into two pieces, depending on whether $m \leq n$ or $m>n$. Clearly, $0 \in I$. It is easy to see that

$$
|I| \leq O\left(1+\max _{0 \leq t \leq 1}|\xi(t)|\right)
$$

for example, by considering the differential equation (2.1) directly. This implies $\mathbf{P}[|I| \geq m] \leq O(1) e^{-C(\kappa) m^{2}}$, for some $C(\kappa)>0$. Since

$$
\left|y \hat{f}_{1}^{\prime}(x+i y)\right| \leq O(1) \operatorname{Im} \hat{f}_{1}(x+i y) \leq O(1)
$$

for $0<y \leq 1$, we have the trivial estimate

$$
\begin{aligned}
\mathbf{E}\left[\sum_{j=-\infty}^{\infty}\left|\hat{f}_{1}^{\prime}\left(z_{j, n}\right) y_{n}\right|^{a} 1_{\mathcal{A}(j, n, m)}\right] & \leq O(1) m 2^{n} \mathbf{P}[|I| \geq m] \\
& \leq O(1) m 2^{n} e^{-C(\kappa) m^{2}}
\end{aligned}
$$

for all $m \geq 0$. This shows that in (8.3) the terms with $m \geq n$ have finite sum. For $m \leq n$, we use Theorem 3.2 together with (3.17) and obtain

$$
\mathbf{E}\left[\sum_{j=-\infty}^{\infty}\left|\hat{f}_{1}^{\prime}\left(z_{j, n}\right) y_{n}\right|^{a} 1_{\mathcal{A}(j, n, m)}\right] \leq C(h, \kappa, b) m^{2 b+1} 2^{-n(\lambda-2 b-1)},
$$

where $a, \lambda, b$ and $\kappa$ are related by (3.6) with $\nu=1$ and $b>0$. For $b$ between $1 / 2$ and $2 / \kappa$ we have $\lambda-2 b-1>0$ and conclude that $\mathbf{E}[\tilde{S}(a)]<\infty$ in this range. By (8.2), $\mathbf{E}[S(a)]<\infty$ for $\kappa<4, a>1+\kappa / 8$ and for $\kappa>4, a>1+2 / \kappa$.

Now consider $a=\delta(\kappa)$. Suppose that $2^{-n}<h / 4$ and $\hat{f}_{1}\left(z_{j, n}\right) \geq h$. We claim that the harmonic measure in $H_{1}$ of $K_{1}$ from $\hat{f}_{1}\left(z_{j, n}\right)$ is bounded from 
zero. Indeed, fix a constant $h_{1}>1$ such that $h_{1} / 2 \geq \sup \left\{\operatorname{Im} z: z \in K_{1}\right\}$. (It is easy to see that $h_{1}=4$ works.) Consider the harmonic function $Y(z):=$ $\operatorname{Im} g_{1}(z)$ on $H_{1}$. Then $Y(x+i y)-y \rightarrow 0$ uniformly in $x$, as $y \rightarrow \infty$, by the hydrodynamic normalization (2.2) of $g_{1}$. Since $\mathbb{H}+\frac{i}{2} h_{1} \subset H_{1}$, the maximum principle applied to $Y(z)-\operatorname{Im} z$ implies that $Y\left(x+i h_{1}\right) \geq h_{1} / 2$ for all $x \in \mathbb{R}$. Consequently, for every $z \in H_{1}$ with $\operatorname{Im} z<h_{1}$, the harmonic measure in $H_{1} \cap\left\{y<h_{1}\right\}$ from $z$ of the line $y=h_{1}$ is at most $2 Y(z) / h_{1}$. However, the harmonic measure from $z$ of $y=h_{1}$ in the domain $\mathbb{H} \cap\left\{y<h_{1}\right\}$ is $\operatorname{Im} z / h_{1}$. Therefore, the harmonic measure from $z$ of $K_{1}$ is at least the difference between these two quantities, which is at least $\operatorname{Im} z / h_{1}-2 Y(z) / h_{1}$. Applying this to $z:=\hat{f}_{1}\left(z_{j, n}\right)$ proves our claim that the harmonic measure of $K_{1}$ from $\hat{f}_{1}\left(z_{j, n}\right)$ in $H_{1}$ is bounded from zero. This implies that the harmonic measure of $I$ from $z_{j, n}$ in $\mathbb{H}$ is bounded from zero, which implies that at bounded hyperbolic distance from $z_{j, n}$ we can find a point $z_{j^{\prime}, n^{\prime}}$ with $x_{j^{\prime}, n^{\prime}} \in I$. Consequently, to prove that $\mathbf{E}\left[\tilde{S}_{h}(\delta(\kappa))\right]=\infty$ for all sufficiently small $h>0$, it suffices to prove that for all $n_{0} \in \mathbb{N}$ and all sufficiently small $h>0$

$$
\mathbf{E}\left[\sum_{j=-\infty}^{\infty} \sum_{n=n_{0}}^{\infty} 1_{\operatorname{Im} \hat{f}_{1}\left(z_{j, n}\right)>h}\left|\hat{f}_{1}^{\prime}\left(z_{j, n}\right) y_{n}\right|^{\delta(\kappa)}\right]=\infty .
$$

This follows from Lemma 3.3 with $b=1 / 2$ if $\kappa<4$ or $b=2 / \kappa$ if $\kappa>4$. An appeal to (8.2) gives $\mathbf{E}[S(\delta(\kappa))]=\infty$. Since $\mathbf{E}[S(a)]<\infty$ for $a>\delta(\kappa)$, it also follows that $\mathbf{E}[S(a)]=\infty$ for $a<\delta(\kappa)$, and the proof is complete.

Corollary 8.5. For all $\kappa>0, \kappa \neq 4$, and all sufficiently small $h>0$

$$
\limsup _{\varepsilon \rightarrow 0}|\log \varepsilon|^{1+h} \varepsilon^{\delta(\kappa)} \mathbf{E}\left[N\left(\varepsilon, \partial K_{1} \cap\{y>h\}\right)\right]=\infty .
$$

Proof. Denote by $W(n)$ the number of $Q \in W$ with $2^{-n-1}<d(Q) \leq 2^{-n}$. Then (8.1) immediately gives

$$
W(n) \leq C N\left(2^{-n}, \partial K_{1} \cap\{y>h\}\right) .
$$

The corollary now follows from $S(a)=\sum_{n=0}^{\infty} W(n) 2^{-a n}$ and Theorem 8.3.

Finally we state an upper bound for the covering dimension.

THEOREM 8.6. For all $\kappa, h>0$ and $a>\delta(\kappa)$, a.s.

$$
\lim _{\varepsilon \rightarrow 0} \varepsilon^{a} N\left(\varepsilon, \partial K_{1} \cap\{y>h\}\right)=0 .
$$

Proof. Suppose first that $\kappa \neq 4$. By Theorem 5.2 we know that $\hat{f}_{1}$ is a.s. Hölder continuous. There is no lower bound version of (8.4) for general domains. However, by results of Pommerenke [Pom92] and Makarov [Mak98] it is known that for Hölder domains (i.e., the conformal map from $\mathbb{U}$ into the 
domain is Hölder) the asymptotics of $N$ can be recovered from the convergence exponent of the Whitney decomposition. Our proof follows this philosophy. We claim that there is a (random) constant $C=C(\omega)>0$, depending on $h$ and the Hölder norm of $\hat{f}_{1}$, such that for every small $\varepsilon>0$ and every point $z_{0} \in \partial K_{1} \cap\{y \geq 2 h\}$

$$
\exists Q \in W, \quad Q \subset D\left(z_{0}, \varepsilon\right), \quad \varepsilon \geq d(Q) \geq C \varepsilon /|\log \varepsilon| .
$$

In fact, this follows from [KR97], but we include a short proof here, for the convenience of the reader.

Let $x_{0} \in \mathbb{R}$ be a point such that $\hat{f}_{1}\left(x_{0}\right)=z_{0}$. Clearly, there is such an $x_{0}$, because $\hat{f}_{1}$ extends continuously to $\overline{\mathbb{H}}$. Let $\beta(s):=\hat{f}_{1}\left(x_{0}+i s\right), s>0$, let $\hat{Q}(s)$ be the set of Whitney cells $Q \in W$ which intersect $\beta((0, s))$, and set

$$
q(s):=\sum_{Q \in \hat{Q}(s)} d(Q) .
$$

Since $\hat{f}_{1}$ is an isometry between the hyperbolic metric of $\mathbb{H}$ and that of $H_{1}$, it follows that for every $s>0$ the $\operatorname{arc} \beta[s, 2 s]$ meets a bounded number of Whitney cells, and each of these has Euclidean diameter comparable to the Euclidean diameter of $\beta[s, 2 s]$. Suppose that $\hat{f}_{1}$ is Hölder with exponent $\alpha>0$. Then, $d(Q) \leq O(1) s^{\alpha}$ for $Q \in \hat{Q}(s)$, and consequently also $q(s) \leq O(1) s^{\alpha}$. Observe that there is a constant $c_{0}$ so that $q(s) / \sup \left\{q\left(s^{\prime}\right): s^{\prime}<s\right\}<c_{0}$, because there are constant upper bounds on the number of Whitney cells containing a point, and on the ratio $d(Q) / d\left(Q^{\prime}\right)$ for neighboring Whitney cells $Q$ and $Q^{\prime}$. Let $s_{0}:=\sup \{s: q(s)<\varepsilon\}$ and $s_{1}:=\sup \left\{s: q(s)<c_{0}^{-1} \varepsilon / 2\right\}$. The choice of $c_{0}$ ensures that $q\left(s_{0}\right)-q\left(s_{1}\right) \geq \varepsilon / 2$. Clearly $\hat{Q}\left(s_{0}\right) \backslash \hat{Q}\left(s_{1}\right)$ has at most $O\left(1+\log \left(s_{0} / s_{1}\right)\right)$ cells. Therefore, there is some $Q \in \hat{Q}\left(s_{0}\right) \backslash \hat{Q}\left(s_{1}\right)$ with $d(Q) \geq \varepsilon / O\left(1+\log \left(s_{0} / s_{1}\right)\right)$. Since $q(s) \leq O(1) s^{\alpha}$, it follows that $O(1) s_{1} \geq$ $\varepsilon^{1 / \alpha}$, and we get $O(1) d(Q) \geq \varepsilon /|\log \varepsilon|$, proving (8.5).

We know that for every set $F \subset \mathbb{C}$ the covering number $N(\varepsilon, F)$ is bounded by a constant times the maximum number of disjoint disks of radius $\varepsilon$ centered in $F$. Therefore, by (8.5), $N\left(\varepsilon, \partial K_{1} \cap\{y>2 h\}\right)$ is bounded by a constant times the number of Whitney cells in $W$ with size between $O(1) \varepsilon$ and $\varepsilon / O(|\log \varepsilon|)$; that is,

$$
N\left(2^{-n}, \partial K_{1} \cap\{y>2 h\}\right) \leq C(\omega) \sum_{j=n}^{n+O(\log n)} W(j),
$$

which implies for all $a^{\prime}>0$,

$$
N\left(2^{-n}, \partial K_{1} \cap\{y>2 h\}\right) \leq C(\omega) 2^{(n+O(\log n)) a^{\prime}} S\left(a^{\prime}\right) .
$$

By Theorem 8.3, $S\left(a^{\prime}\right)<\infty$ a.s. for $a^{\prime}>\delta(\kappa)$. Therefore, the case $\kappa \neq 4$ in Theorem 8.6 now follows by taking $a^{\prime} \in(\delta(\kappa), a)$. 
The following argument covers not only the case $\kappa=4$, but the whole range $\kappa \in(0,4]$. For such $\kappa$, Theorem 8.1 can be used, since $\partial K_{1}=\gamma[0,1]$ for $\kappa \in(0,4]$. Although Theorem 8.1 discusses the expected number of balls needed to cover $\gamma[0, \infty) \cap A$, where $A$ is an arbitrary bounded set satisfying $\bar{A} \subset \mathbb{H}$, one can refine the result to bound $N\left(\varepsilon, \partial K_{1} \cap\{y>H\}\right)$, as follows. Given a point $z=x+i y, y>h$, we need to estimate the probability that $z$ is close to $K_{1}$. We may certainly restrict attention to the case where $y$ is bounded by some constant. There is a constant $C \geq 1$ such that the probability that $z$ is close to $K_{1}$ is bounded by the probability that there is some time $t_{1}<1$ such that $\xi\left(t_{1}\right)$ is within distance $C$ from $x$ times the probability that $Z(z)$ is large, conditioned on the existence of such a $t_{1}$. The proof is then completed by applying the estimates in the proof of Theorem 8.1 and the Markov property. The details are left to the reader.

The upper bound for the Hausdorff dimension follows immediately:

Corollary 8.7. For $\kappa>4$, the Hausdorff dimension of $\partial K_{1}$ is a.s. at most $1+2 / \kappa$.

\section{Further discussion and some open problems}

We believe Theorem 5.1 to hold also in the case $\kappa=8$ :

Conjecture 9.1. The $\mathrm{SLE}_{8}$ trace is a continuous path a.s.

Update. This is proved in [LSW].

ConjeCture 9.2. The Hausdorff dimension of the $\mathrm{SLE}_{\kappa}$ trace is a.s. $1+\kappa / 8$ when $\kappa<8$.

Update. A proof of this result is announced in [Bef] for $\kappa \neq 4$.

Corollary 8.2 shows that the Hausdorff dimension cannot be larger than $1+\kappa / 8$.

Conjecture 9.3 (Richard Kenyon). The Hausdorff dimension of $\partial K_{1}$ is a.s. $1+2 / \kappa$ when $\kappa \geq 4$.

Corollary 8.7 shows that the Hausdorff dimension cannot be larger than $1+2 / \kappa$.

Problem 9.4. Find an estimate for the modulus of continuity for the trace of $\mathrm{SLE}_{4}$. Improve the estimate for the modulus of continuity for $\mathrm{SLE}_{\kappa}$ for other $\kappa$. What better modulus of continuity can be obtained with a different parametrization of the trace? 


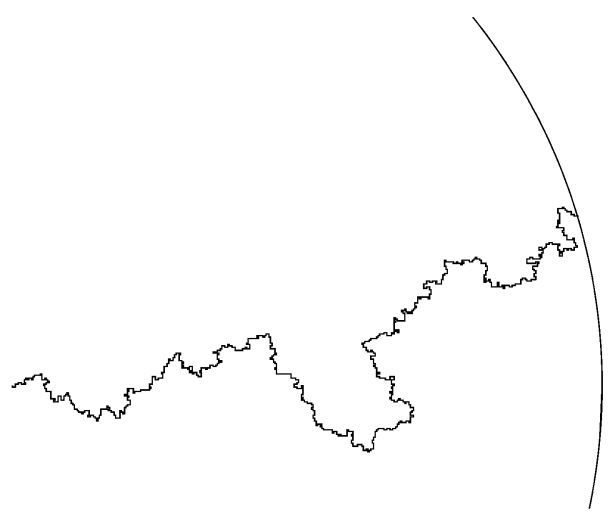

Figure 9.1: A sample of the loop-erased random walk; believed to converge to radial $\mathrm{SLE}_{2}$.

Based on the conjectures for the dimensions [Dup00], Duplantier suggested that $\mathrm{SLE}_{16 / \kappa}$ describes the boundary of the hull of $\mathrm{SLE}_{\kappa}$ when $\kappa>4$. This has further support, some of which is discussed below. However, at this point there isn't even a precise version for this duality statement.

Problem 9.5. Understand the relation between the boundary of the $\mathrm{SLE}_{\kappa}$ hull and $\mathrm{SLE}_{16 / \kappa}$ when $\kappa>4$.

Update. See [Dub] for recent progress on this problem.

We now list some conjectures about discrete processes tending to $\mathrm{SLE}_{\kappa}$ for various $\kappa$. Let $D$ be a bounded simply connected domain in $\mathbb{C}$ with smooth boundary, and let $a$ and $b$ be two distinct points in $\partial D$. Let $\phi$ be a conformal map from $\mathbb{H}$ to $D$ such that $\phi(0)=a$ and $\phi(\infty)=b$. The SLE trace in $D$ from $a$ to $b$ is defined as the image under $\phi$ of the trace of chordal SLE in $\mathbb{H}$. Similarly, one defines radial SLE in arbitrary simply connected domains strictly contained in $\mathbb{C}$.

In [Sch00] it was shown that if the loop-erased random walk in simply connected domains in $\mathbb{C}$ has a conformally invariant scaling limit, then this must be radial $\mathrm{SLE}_{2}$. See Figure 9.1 for a sample of the loop-erased random walk in $\mathbb{U}$.

In [Sch00] it was also conjectured that the UST Peano curve, with appropriate boundary conditions (see Figure 9.2), tends to $\mathrm{SLE}_{8}$ as the mesh goes to zero. This offers additional support to Duplantier's conjectured duality 9.5 , Since the outer boundary of the hull of the UST Peano curve consists of two loop-erased random walks, one in the tree and one in the dual tree. See [Sch00] for additional details. 


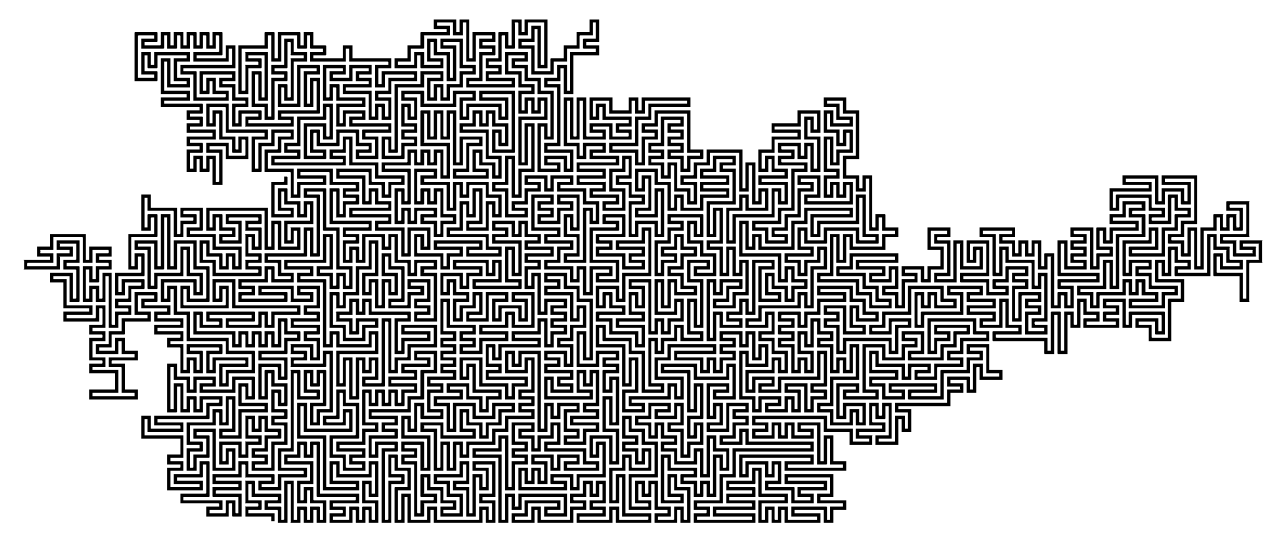

Figure 9.2: A piece of the UST Peano curve; believed to converge to SLE . $_{\text {. }}$

Update. The convergence of LERW to $\mathrm{SLE}_{2}$ and of the UST Peano path to $\mathrm{SLE}_{8}$ is proved in [LSW].

Conjecture 9.6. Consider a square grid of mesh $\varepsilon$ in the unit disk $\mathbb{U}$. Then the uniform measure on simple grid paths from 0 to $\partial \mathbb{U}$ on this grid converges weakly to the trace of radial $\mathrm{SLE}_{8}$ as $\varepsilon \searrow 0$, up to reparametrization. The uniform measure on simple paths joining two distinct fixed boundary points converges weakly to the trace of chordal $\mathrm{SLE}_{8}$, up to reparametrization.

We have not bothered to make this conjecture entirely precise, since there is more than one natural choice for the topology on path space. However, this does not seem to be very important at this point.

Note that we do not require that the grid path be space filling (i.e., visit every vertex). Indeed, it is not hard to see that a uniformly selected path will be essentially filling, in the sense that the probability that a nonempty open set inside $\mathbb{U}$ is unvisited goes to zero as $\varepsilon \searrow 0$.

Consider an $n \times n$ grid in the unit square, and identify the vertices of the right and bottom boundary arcs to a single vertex $w_{0}$. Let $G_{n}$ denote this graph, and note that the dual graph $G_{n}^{\dagger}$ is obtained by rotating $G_{n} 180^{\circ}$ and shifting appropriately. See Figure 9.3. Let $\omega$ be a sample from the critical random-cluster measure on $G_{n}$ with parameters $q$ and $p=p(q)=\sqrt{q} /(1+\sqrt{q})$ (which is the conjectured value of $p_{c}=p_{c}(q)$ ) and let $\omega^{\dagger}$ be the set of edges in $G_{n}^{\dagger}$ that do not cross edges of $\omega$. Then the law of $\omega^{\dagger}$ is the random-cluster measure on $G_{n}^{\dagger}$ with the same parameters as for $\omega$. Let $\beta$ be the set of points in the unit square that are at equal distance from the component of $\omega$ containing $w_{0}$ and the component of $\omega^{\dagger}$ containing the wired vertex of $G_{n}^{\dagger}$. 


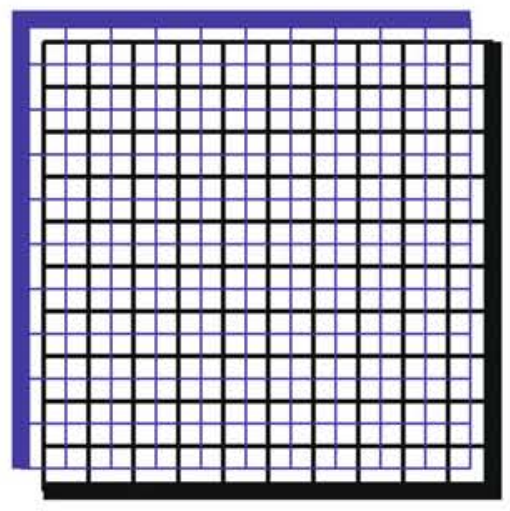

Figure 9.3: A partially wired grid and its dual.

Conjecture 9.7. If $q \in(0,4)$, then as $n \rightarrow \infty$, the law of $\beta$ converges weakly to the trace of $\mathrm{SLE}_{\kappa}$, up to reparametrization, where $\kappa=\frac{4 \pi}{\cos ^{-1}(-\sqrt{q} / 2)}$.

The above value of $\kappa$ is derived by applying the predictions [SD87] of the dimensions of the perimeters of clusters in the random cluster model.

Consider a random-uniform domino tiling of the unit square, such as in the top right of Figure 9.4. Taking the union with another independent domino tiling with the two lower corner squares removed, one gets a path joining the two removed squares; see Figure 9.4. This model was considered by [RHA97]. Richard Kenyon proposed the following

Problem 9.8. Does this double-domino model converge weakly (up to reparametrization) to $\mathrm{SLE}_{4}$ as the mesh of the grid tends to zero?

Kenyon also produced some calculations for this model that agree with the corresponding calculations for $\mathrm{SLE}_{4}$.

Let $a \in \mathbb{R}, \varepsilon>0$, and consider the following random walk $S$ on the grid $\varepsilon \mathbb{Z}^{2}$. Set $S_{0}=0$, and inductively, we construct $S_{n+1}$ given $\left\{S_{0}, \ldots, S_{n}\right\}$. If $S_{n} \notin \mathbb{U}$, then stop. Otherwise, let $h_{n}: \varepsilon \mathbb{Z}^{2} \rightarrow[0,1]$ be the function which is 1 outside of $\mathbb{U}, 0$ on $\left\{S_{0}, \ldots, S_{n}\right\}$, and discrete-harmonic elsewhere. Now choose $S_{n+1}$ among the neighbors $v$ of $S_{n}$ such that $h(v)>0$, with probability proportional to $h(v)^{a}$. When $a=1$, this is called Laplacian random walk, and is known to be equivalent to loop-erased random walk. When $a=0$ this walk is believed to be closely related to the boundary of percolation clusters.

The following problem came up in discussions with Richard Kenyon.

Problem 9.9. For what values of $a$ does this process converge weakly to radial SLE? What is the correspondence $\kappa=\kappa(a)$ ? 


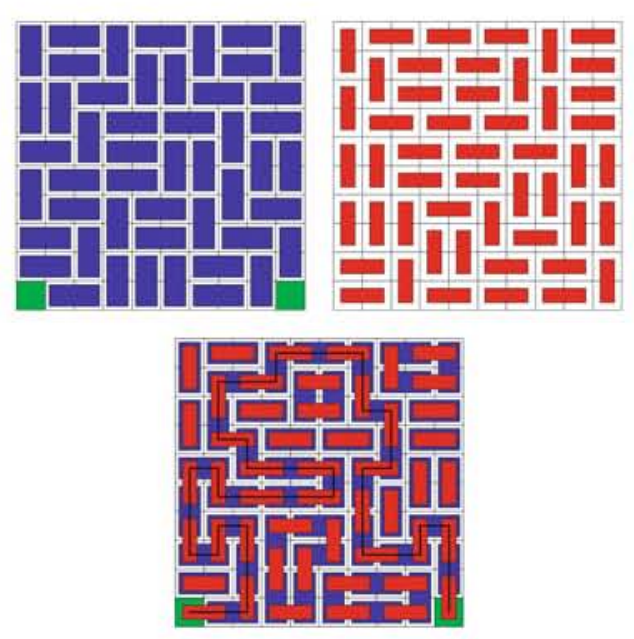

Figure 9.4: The double domino path.

Motivated in part by Duplantier's duality conjecture 9.5, in [LSW03] it is conjectured that chordal $\mathrm{SLE}_{8 / 3}$ is the scaling limit of the self-avoiding walk in a half-plane. (That is, the limit as $\delta \searrow 0$ of the limit as $n \nearrow \infty$ of the law of a random-uniform $n$-step simple path from 0 in $\delta \mathbb{Z}^{2} \cap \overline{\mathbb{H}}$.) Some strong support to this conjecture is provided there, which in turn can be viewed as further support for the duality in 9.5.

Many of the processes believed to converge to chordal SLE have the following reversibility property. Up to reparametrization, the path from $a$ to $b$ in $D(a, b \in \partial D)$ has the same distribution as the path from $b$ to $a$. It is then reasonable to conjecture:

CONJECTURE 9.10. The chordal SLE trace is reversible for $\kappa \in[0,8]$.

It is not too hard to see that this fails for $\kappa>8$. In particular, when $\kappa>8$ the chordal SLE path is more likely to visit 1 before visiting $i$. Since inversion in the unit circle preserves 1 and $i$, the probability for visiting $i$ before 1 would have to be $1 / 2$ if one assumes reversibility. To apply this argument one has to note that a.s. the path visits 1 and $i$ exactly once.

Update. The above conjecture is known to hold when $\kappa=0,2,8 / 3,6,8$. The cases $\kappa=2,8$ follow from [LSW] (though some additional work is needed in the case where $\kappa=2$, since the convergence of the LERW is to radial SLE), the case $\kappa=6$ follows from Smirnov's Theorem [Smi01], and the case $\kappa=$ $8 / 3$ follows from the characterization in [LSW03] of $\mathrm{SLE}_{8 / 3}$ as the restriction measure with exponent $5 / 8$. 
Some conjectures and simulations concerning the winding numbers of SLE's are described in [WW].

Acknowledgments. The authors thank Richard Kenyon for useful discussions and Jeff Steiff for numerous comments on a previous version of this paper.

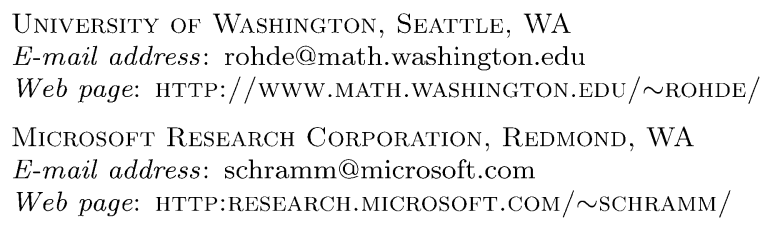

\section{REFERENCES}

[Ahl73] L. V. Ahlfors, Conformal Invariants: Topics in Geometric Function Theory, McGraw-Hill Series in Higher Mathematics, McGraw-Hill Book Co., New York, 1973.

[Bef] V. Beffara, The dimension of the SLE curves; arXiv:math.PR/0211322.

[Car] J. CARDY, Conformal invariance and percolation; arXiv:math-ph/0103018.

[CM01] L. Carleson and N. Makarov, Aggregation in the plane and Loewner's equation, Comm. Math. Phys. 216 (2001), 583-607.

[Dub] J. Dubedat, $\operatorname{SLE}(\kappa, \rho)$ martingales and duality; arXiv:math.PR/0303128.

[Dup00] B. Duplantier, Conformally invariant fractals and potential theory, Phys. Rev. Lett., 84 (2000), 1363-1367.

[Dur96] R. Durrett, Stochastic Calculus: A Practical Introduction, CRC Press, Boca Raton, FL, 1996.

[emot53] A. Erdélyi, W. Magnus, F. Oberhettinger, and F. G. Tricomi, Higher Transcendental Functions Vol. I. Based, in part, on notes left by Harry Bateman. McGraw-Hill Book Company, Inc., New York, 1953.

[JM95] P. W. Jones and N. G. Makarov, Density properties of harmonic measure, Ann. of Math. 142(1995), 427-455.

[KR97] P. Koskela and S. Rohde, Hausdorff dimension and mean porosity, Math. Ann. 309 (1997), 593-609.

[LSW01a] G. F. LAWLer, O. Schramm, and W. Werner, Values of Brownian intersection exponents. I. Half-plane exponents, Acta Math. 187 (2001), 237-273.

[LSW01b] , Values of Brownian intersection exponents. II. Plane exponents, Acta Math. 187 (2001), 275-308.

[LSW02] - Values of Brownian intersection exponents. III. Two-sided exponents, Ann. Inst. H. Poincaré Probab. Statist. 38 (2002), 109-123.

[LSW03] , Conformal restriction: the chordal case, J. Amer. Math. Soc. 16 (2003), 917-955 (electronic).

[LSW] - Conformal invariance of planar loop-erased random walks and uniform spanning trees, Ann. Probab. 32 (2004), 939-995.

[Mak98] N. G. Makarov, Fine structure of harmonic measure, Algebra i Analiz 10 (1998), $1-62$. 
[MR] D. Marshall and S. Rohde, The Loewner differential equation, preprint.

[Pom92] Сн. Pommerenke, Boundary Behaviour of Conformal Maps, Springer-Verlag, New York, 1992.

[RHA97] R. Raghavan, C. L. Henley, and S. L. Arouh, New two-color dimer models with critical ground states, J. Statist. Phys. 86 (1997), 517-550.

[RY99] D. Revuz and M. Yor, Continuous martingales and Brownian motion, Third edition, Grundlehren der Mathematischen Wissenschaften, Fundamental Principles of Mathematical Sciences 293, Springer-Verlag, New York, 1999.

[Sch00] O. SснRAmm, Scaling limits of loop-erased random walks and uniform spanning trees, Israel J. Math. 118 (2000), 221-288.

[SD87] H. SAleur and B. Duplantier, Exact determination of the percolation hull exponent in two dimensions, Phys. Rev. Lett. 58 (1987), 2325-2328.

[Smi01] S. Smirnov, Critical percolation in the plane: conformal invariance, Cardy's formula, scaling limits, C. R. Acad. Sci. Paris Sér. I Math. 333 (2001), 239-244.

[SS] O. Schramm and S. ShefField, The harmonic explorer and its convergence to SLE(4); arXiv:math.PR/0310210.

[Wer01] W. Werner, Critical exponents, conformal invariance and planar Brownian motion, in European Congress of Mathematics, Vol. II (Barcelona, 2000), Progr. Math. 202, 87-103, Birkhäuser, Basel, 2001.

[WW] B. WeILAND and D. B. Wilson, Winding angle variance of Fortuin-Kasteleyn contours, Phys. Rev. E, to appear.

(Received August 8, 2001) 